\title{
Taille des valeurs de fonctions $L$ de carrés symétriques au bord de la bande critique
}

Emmanuel Royer and Jie Wu

\begin{abstract}
For each weight $k$ and level $N$ square free and without small prime factors, we prove the existence of primitive forms $f_{+}$and $f_{-}$of weight $k$ and level $N$ such that

$$
L\left(1, \operatorname{sym}^{2} f_{+}\right) \gg_{k}[\log \log (3 N)]^{3}
$$

and

$$
L\left(1, \operatorname{sym}^{2} f_{-}\right) \ll_{k}[\log \log (3 N)]^{-1} .
$$

The result comes from a delicate study of the moments of $L\left(1, \operatorname{sym}^{2} f\right)$. This study gives also results for squarefree levels but with small prime factors. It provides counterexamples to the equivalence between harmonic and natural means.
\end{abstract}

\section{Introduction}

Les valeurs au bord de la bande critique des fonctions $L$ contiennent des informations algébriques intéressantes. Par exemple, considérons $\mathcal{K}_{n}$ l'ensemble des corps totalement réels de degré $n$ et de clôture algébrique ayant le groupe symétrique $S_{n}$ pour groupe de Galois. Si $K \in \mathcal{K}_{n}$, on note $h(K)$ le nombre de classes d'idéaux de $K$ et $d(K)$ son discriminant. En étudiant la quantité $L\left(1, \chi_{K}\right)$, où $L\left(s, \chi_{K}\right)$ est la fonction $L$ d'Artin associée à $K$, on obtient des renseignements sur la taille de $h(K)$. Admettant que, pour tout $K \in \mathcal{K}_{n}$, la fonction $L\left(s, \chi_{K}\right)$ est entière et satisfait à l'hypothèse de Riemann, une méthode due à Littlewood, jointe à des travaux de Remak,

2000 Mathematics Subject Classification : 11F12, 11F25, 11F67, 11M41, 11N36, $11 \mathrm{~N} 37$. Keywords : forme automorphe, carré symétrique, fonction $L$, valeur spéciale. 
permet de prouver l'existence, pour tout $n \geq 2$, d'un réel $\beta_{n}>0$ tel que, pour tout $K \in \mathcal{K}_{n}$, on a

$$
h(K) \leq \beta_{n} \sqrt{d(K)}\left[\frac{\log \log d(K)}{\log d(K)}\right]^{n-1} .
$$

Récemment, Duke [3] a montré l'optimalité (à la constante $\beta_{n}$ près) de cette majoration. Sous les mêmes hypothèses, il montre l'existence, pour tout $n \geq 2$, d'un réel $\alpha_{n}>0$ tel qu'il existe $K \in \mathcal{K}_{n}$ vérifiant

$$
h(K) \geq \alpha_{n} \sqrt{d(K)}\left[\frac{\log \log d(K)}{\log d(K)}\right]^{n-1} .
$$

Dans cet article, on étudie la valeur au bord de la bande critique de la fonction $L\left(s, \operatorname{sym}^{2} f\right)$ de carré symétrique d'une forme modulaire. Contrairement à ce qui se passe pour la fonction $L$ d'une forme modulaire, la valeur au bord de la bande critique de cette fonction introduite par Shimura a d'importants liens avec la théorie géométrique des nombres : ainsi, elle est reliée au degré des paramétrisations modulaires (voir par exemple [4]). Si $k>0$ est un entier pair et $N>0$ un entier, on note $\mathrm{H}_{k}^{*}(N)$ l'ensemble des formes primitives de poids $k$ sur $\Gamma_{0}(N)$. La meilleure estimation actuelle pour $L\left(1, \operatorname{sym}^{2} f\right)$ est due à Goldfeld, Hoffstein et Lieman qui démontrent, dans [5], l'existence d'un réel $\alpha>0$ tel que, pour tout entier $k>0$ pair, tout entier $N>0$ sans facteur carré et toute forme $f \in \mathrm{H}_{k}^{*}(N)$ on a

$$
L\left(1, \operatorname{sym}^{2} f\right)+L\left(1, \operatorname{sym}^{2} f\right)^{-1} \leq \alpha \log (k N) .
$$

Notre but est de tester la majoration (1.1), c'est-à-dire de trouver une fonction $F$ (resp. $G$ ) la plus grande (resp. petite) possible telle que, pour tout entier $N$ sans facteur carré, il existe des formes primitives $f_{+}$et $f_{-}$de niveau $N$ telles que

$$
L\left(1, \operatorname{sym}^{2} f_{+}\right) \geq F(N) \quad \text { et } \quad L\left(1, \operatorname{sym}^{2} f_{-}\right) \leq G(N) .
$$

Désignons par $P^{-}(N)$ le plus petit facteur premier de $N$ et $\mu$ la fonction de Möbius. En poursuivant l'étude entamée dans [18] on montre le

Théorème A. Soit

$$
\mathcal{N}_{\text {cri }}:=\left\{N \in \mathbb{N}^{*}: \mu(N) \neq 0 \text { et } P^{-}(N) \geq \log (3 N)\right\} .
$$

Pour tous $k>0$ entier pair, $N$ entier sans facteur carré et $C>0$ réel, on introduit

$$
\mathrm{H}_{k}^{*+}\left(N ; C, \operatorname{sym}^{2}\right)=\left\{f \in \mathrm{H}_{k}^{*}(N): L\left(1, \operatorname{sym}^{2} f\right) \geq C[\log \log (3 N)]^{3}\right\}
$$

et

$$
\mathrm{H}_{k}^{*-}\left(N ; C, \operatorname{sym}^{2}\right)=\left\{f \in \mathrm{H}_{k}^{*}(N): L\left(1, \operatorname{sym}^{2} f\right) \leq C[\log \log (3 N)]^{-1}\right\} .
$$


Alors, pour tout $\varepsilon>0$, il existe deux réels $C>0$ et $\xi>0$ ne dépendant que de $\varepsilon$ et $k$ tels que pour tout $N \in \mathcal{N}_{\text {cri }}$ on a

$$
\# \mathrm{H}_{k}^{* \pm}\left(N ; C, \operatorname{sym}^{2}\right) \geq e^{-\xi[\log (3 N)]^{\varepsilon}} \# \mathrm{H}_{k}^{*}(N) .
$$

En particulier, il existe un réel $K>0$ ne dépendant que de $k$ tel que, pour tout $N \in \mathcal{N}_{\text {cri }}$, il existe $f_{+} \in \mathrm{H}_{k}^{*}(N)$ et $f_{-} \in \mathrm{H}_{k}^{*}(N)$ vérifiant

$$
L\left(1, \operatorname{sym}^{2} f_{+}\right) \geq K[\log \log (3 N)]^{3} \quad \text { et } \quad L\left(1, \operatorname{sym}^{2} f_{-}\right) \leq K[\log \log (3 N)]^{-1} \text {. }
$$

Remarque A. On montre par ailleurs (en admettant l'hypothèse de Riemann généralisée) que ce résultat est optimal. Plus précisément, si $N$ est un entier sans facteur carré et si $f \in \mathrm{H}_{k}^{*}(N)$, l'hypothèse de Riemann pour $L\left(s, \operatorname{sym}^{2} f\right)$ implique

$$
[\log \log (3 N)]^{-1} \ll L\left(1, \operatorname{sym}^{2} f\right) \ll[\log \log (3 N)]^{3} .
$$

D'autre part, si $N_{j}=p_{1} \cdots p_{j}$ est le produit des $j$ premiers nombres premiers, l'hypothèse de Riemann pour $L\left(s, \operatorname{sym}^{2} f\right)$ implique

$$
1 \ll L\left(1, \operatorname{sym}^{2} f\right) \ll 1 .
$$

L'équation (1.2) montre l'optimalité des deux inégalités du théorème A. L'équation (1.3) montre que la restriction à $N \in \mathcal{N}_{\text {cri }}$ est indispensable. Il est à noter que les propriétés arithmétiques de $N$ jouent un rôle fondamental dans la variation des valeurs de $L\left(1, \operatorname{sym}^{2} f\right)$ lorsque $f$ parcourt $\mathrm{H}_{k}^{*}(N)$.

Remarque B. Dans [24], Zagier prouve que, pour tout entier $N \geq 1$, il existe un entier $q \geq N$ et une forme primitive $f$ de niveau $q$ tels que $\langle f, f\rangle \gg q$. On améliore partiellement ce résultat puisque, compte-tenu de (3.18) et (3.2) notre résultat s'énonce : pour tout entier $N \in \mathcal{N}_{\text {cri }}$, il existe $f \in \mathrm{H}_{k}^{*}(N)$ telle que $\langle f, f\rangle \gg N[\log \log (3 N)]^{3}$. Cependant, notre méthode ne donne rien pour les niveaux avec facteur carré alors que la méthode de Zagier utilise au contraire des formes de niveau avec facteur carré.

Pour établir le théorème $\mathrm{A}$, on calcule une formule asymptotique des moments harmoniques de $L\left(1, \operatorname{sym}^{2} f\right)$ :

$$
\sum_{f \in \mathrm{H}_{k}^{*}(N)} \omega(f) L\left(1, \operatorname{sym}^{2} f\right)^{ \pm \ell}
$$

pour tout $\ell \in \mathbb{N}^{*}$. Le facteur $\omega(f)$ est le facteur harmonique habituel dans cette théorie $-c f$. (3.2).

La formule asymptotique donnée dans le théorème $\mathrm{A}$ de [18] est insuffisante. Une analyse minutieuse des dépendances en tous les paramètres est nécessaire. Cette analyse exige un changement radical des techniques utilisées pour évaluer les termes d'erreurs de [18]. 
Par ailleurs, notre formule asymptotique (voir le théorème B ci-dessous), valable uniformément pour tous les paramètres (à l'exception du poids $k$ des formes modulaires), porte plus d'informations que celle de [18]. En particulier si la seule contrainte sur le niveau $N$ est qu'il est sans facteur carré, alors les moments positifs et négatifs n'existent pas.

Avant d'énoncer notre formule asymptotique pour (1.4), il est nécessaire de présenter certaines notations. Les lettres grasses telles que $\mathbf{a}, \mathbf{b}, \mathbf{c}, \ldots$ désignent des vecteurs. Leurs coordonnées sont numérotées en indices, ainsi $\mathbf{a}=\left(a_{1}, \ldots, a_{m}\right)$. Le déterminant (noté $\left.|\cdot|\right)$ d'un vecteur est le produit de ses coordonnées, ainsi $|\mathbf{a}|=a_{1} \cdots a_{m}$. Le produit de deux vecteurs est le vecteur de même dimension obtenu en multipliant entre elles les coordonnées de même indice, ainsi $\mathbf{a b}=\left(a_{1} b_{1}, \ldots, a_{m} b_{m}\right)$. On désigne par $\mathbb{1}^{(N)}-\operatorname{resp}$. $\mathbb{1}_{N}$ la fonction caractéristique des entiers premiers à $N$-resp. ayant mêmes diviseurs premiers que $N-c f$. (2.1) et (2.2). Pour tout $\mathbf{b} \in \mathbb{N}^{\ell}$, on définit l'ensemble

$$
\mathcal{E}_{\ell}(\mathbf{b}):=\left\{\mathbf{d} \in \mathbb{N}^{\ell-1}: d_{j} \mid\left(\frac{b_{1} \cdots b_{j}}{d_{1} \cdots d_{j-1}}, b_{j+1}\right)^{2} \quad(1 \leq j \leq \ell-1)\right\} .
$$

On fait les conventions suivantes : $\mathbb{N}^{0}:=\{1\}, \mathcal{E}_{1}(\mathbf{b}):=\{1\}$ et $d_{1} \cdots d_{0}:=1$. Pour tout entier $n \in \mathbb{N}^{*}$, on pose ensuite

$$
m_{+\ell}^{(N)}(n):=\sum_{\substack{\mathbf{a}, \mathbf{b}, \mathbf{c} \in \mathbb{N}^{\ell} \\\left|\mathbf{a}^{2} \mathbf{b} \mathbf{c}\right|=n}} \mathbb{1}^{(N)}(|\mathbf{a b}|) \frac{\mathbb{1}_{N}(|\mathbf{c}|)}{|\mathbf{c}|} \sum_{\substack{\mathbf{d} \in \mathcal{E}_{\ell}(\mathbf{b}) \\|\mathbf{d}|=|\mathbf{b}|}} 1
$$

et

$$
m_{-\ell}^{(N)}(n):=\sum_{\substack{\mathbf{a}, \mathbf{b}, \mathbf{c}, \mathbf{e} \in \mathbb{N}^{\ell} \\\left|\mathbf{a} \mathbf{b}^{2} \mathbf{c}^{3} \mathbf{e}\right|=n}} \mathbb{1}^{(N)}(|\mathbf{a b c}|) \frac{\mathbb{1}_{N}(|\mathbf{e}|)}{|\mathbf{e}|} \prod_{j=1}^{\ell} \mu\left(a_{j} b_{j} c_{j}\right) \mu\left(b_{j}\right) \mu\left(e_{j}\right) \sum_{\substack{\mathbf{d} \in \mathcal{E}_{\ell}(\mathbf{a b}) \\|\mathbf{d}|=|\mathbf{a b}|}} 1 .
$$

Enfin on définit

$$
M_{ \pm \ell}^{(N)}:=\sum_{n=1}^{\infty} \frac{m_{ \pm \ell}^{(N)}(n)}{n}
$$

et

$$
M_{ \pm \ell}:=\prod_{p} \sum_{\nu \geq 0} \frac{m_{ \pm \ell, \nu}}{p^{\nu}}
$$

où $m_{ \pm \ell, \nu}:=m_{ \pm \ell}^{(N)}\left(p^{\nu}\right)$ pour $p \nmid N$ ne dépend ni de $p$ ni de $N$. Il y a ici une légère différence de notation avec [18]. En notant $M_{ \pm \ell}^{\mathrm{r}}$ ce qui y était noté $M_{ \pm \ell}$, on a

$$
M_{ \pm \ell}=\zeta(2) M_{ \pm \ell}^{\mathrm{r}}
$$


On note $\varphi$ la fonction d'Euler. Notre résultat central est le suivant.

Théorème B. Soit $k>0$ un entier pair. Il existe un réel $\xi>0$ tel que, pour tout entier $N$ sans facteur carré et tout $\ell \in \mathbb{N}^{*}$, on a

$$
\sum_{f \in \mathrm{H}_{k}^{*}(N)} \omega(f) L\left(1, \operatorname{sym}^{2} f\right)^{ \pm \ell}=\frac{\varphi(N)}{N} M_{ \pm \ell}^{(N)}+O_{k}\left(N^{-1 / 13} e^{\xi \ell[\log (2 N)]^{1 / 2}+\xi \ell^{2}}\right)
$$

où la constante impliquée dans le symbole $O$ ne dépend que de $k$.

Si l'on veut déduire du théorème B les valeurs extrêmes de $L\left(1, \operatorname{sym}^{2} f\right)$, il est souhaitable de chercher les valeurs maximales de $\varphi(N) N^{-1} M_{ \pm \ell}^{(N)}$ quand $N$ parcourt un certain ensemble. Le résultat suivant est une précision des théorèmes $\mathrm{A}$ et $\mathrm{C}$ de [18].

Corollaire A. Soit $k>0$ un entier pair. Il existe un réel $\xi>0$ tel que, pour tous $N \in \mathcal{N}_{\text {cri }}$ et $\ell \in \mathbb{N}^{*}$, on a

$$
\begin{aligned}
\sum_{f \in \mathrm{H}_{k}^{*}(N)} \omega(f) L\left(1, \operatorname{sym}^{2} f\right)^{ \pm \ell}=M_{ \pm \ell}[1+O( & \left.\left.\frac{1}{\log \log (3 N)}\right)\right] \\
& +O_{k}\left(N^{-1 / 13} e^{\xi \ell[\log (2 N)]^{1 / 2}+\xi \ell^{2}}\right)
\end{aligned}
$$

où la constante impliquée dans le symbole $O$ ne dépend que de $k$.

La taille de $\varphi(N) N^{-1} M_{ \pm \ell}^{(N)}$ et l'existence de moments dépendent des propriétés arithmétiques de $N$. Pour tout entier $j \geq 1$, on définit $N_{j}:=p_{1} \cdots p_{j}$ où $\left\{p_{j}\right\}_{j \geq 1}$ est la suite strictement croissante des nombres premiers. L'ensemble des entiers $N_{j}$ lorsque $j$ parcourt $\mathbb{N}^{*}$ est noté $\mathcal{N}_{\text {fri }}$. On prouve alors le

Corollaire B. La suite de moments $\left\{\varphi(N) N^{-1} M_{ \pm \ell}^{(N)}\right\}_{N \geq 1, \mu(N) \neq 0}$ n'a pas de limite lorsque $N \rightarrow \infty$. En particulier,

$$
\liminf _{\substack{N \rightarrow+\infty \\ \mu(N) \neq 0}} \frac{\varphi(N)}{N} M_{ \pm \ell}^{(N)}=\lim _{\substack{N \rightarrow+\infty \\ N \in \mathcal{N}_{\text {fri }}}} \frac{\varphi(N)}{N} M_{ \pm \ell}^{(N)}=0
$$

et

$$
\begin{aligned}
& \limsup _{\substack{N \rightarrow+\infty \\
\mu(N) \neq 0}} \frac{\varphi(N)}{N} M_{+\ell}^{(N)}=e^{3 \ell \log \log \ell+O(\ell)}, \\
& \limsup _{\substack{N \rightarrow+\infty \\
\mu(N) \neq 0}} \frac{\varphi(N)}{N} M_{-\ell}^{(N)}=e^{\ell \log \log \ell+O(\ell)} .
\end{aligned}
$$

De plus, pour les moments positifs, on a l'égalité

$$
\limsup _{\substack{N \rightarrow+\infty \\ \mu(N) \neq 0}} \frac{\varphi(N)}{N} M_{+\ell}^{(N)}=M_{+\ell}
$$


Dans [19], le premier auteur a donné une interprétation des moments négatifs en termes combinatoires via les nombres de Riordan. Il en déduit

$$
\log M_{-\ell}=\ell \log \log \ell+\left[\gamma^{*}-2 \log \zeta(2)\right] \ell+O\left(\frac{\ell}{\log \ell}\right) .
$$

Par la suite, le premier auteur et Habsieger [6] ont montré en utilisant de nouveau des techniques combinatoires

$$
\log M_{+\ell}=3 \ell \log \log \ell+3 \gamma^{*} \ell+O\left(\frac{\ell}{\log \ell}\right) .
$$

Dans (1.7) et (1.8), on a noté $\gamma^{*}$ la constante d'Euler. Le corollaire B montre donc que les moments asymptotiques $M_{ \pm \ell}$ sont, à une erreur négligeable près, les limites supérieures des moments $M_{ \pm \ell}^{(N)}$.

Le corollaire B permet aussi d'affiner l'étude du lien entre la moyenne harmonique et la moyenne naturelle. On considère une application $\alpha: \mathrm{H}_{2}^{*}(N) \rightarrow \mathbb{C}$ et on suppose qu'il existe des constantes $A>0, C>0$ et $\delta>0$ telles que, pour tout $N$ premier assez grand on a

$$
\sum_{f \in \mathrm{H}_{2}^{*}(N)} \omega(f)|\alpha(f)| \leq C(\log N)^{A}
$$

et

$$
\max _{f \in \mathrm{H}_{2}^{*}(N)}|\omega(f) \alpha(f)| \leq C N^{-\delta} .
$$

Kowalski \& Michel ont prouvé dans [11, Proposition 2] l'existence de $\gamma>0$ tel que

$$
\frac{1}{\# \mathrm{H}_{2}^{*}(N)} \sum_{f \in \mathrm{H}_{2}^{*}(N)} \alpha(f)=\frac{1}{\zeta(2)} \sum_{f \in \mathrm{H}_{2}^{*}(N)} \omega(f) L\left(1, \operatorname{sym}^{2} f\right) \alpha(f)+O\left(N^{-\gamma}\right) .
$$

S'il fait peu de doute que ce résultat s'étende à $\mathrm{H}_{k}^{*}(N)$ pour tout $k \geq 2$ pair et tout $N \geq 1$ sans facteur carré et sans petit facteur premier, l'absence de petit facteur premier est nécessaire. Pour le voir, on fixe $\ell \in \mathbb{N}, k \geq 2$ pair et on considère comme fonction $\alpha$ l'une des applications

$$
\begin{array}{rlrc}
\alpha_{ \pm \ell}: \mathrm{H}_{k}^{*}(N) & \rightarrow & \mathbb{C} \\
f & \mapsto L\left(1, \mathrm{sym}^{2} f\right)^{ \pm \ell} .
\end{array}
$$

La condition (1.9) est vérifiée grâce au théorème B et au corollaire B et la condition (1.10) est vérifiée grâce aux équations (1.1) et (3.19). Le théorème $\mathrm{B}$, compte-tenu de $(3.18),(3.4)$ et (4.76) conduit donc à

$$
\lim _{\substack{N \rightarrow \infty \\ N \in \mathcal{N}_{\text {fri }}}} \frac{1}{\# \mathrm{H}_{k}^{*}(N)} \sum_{f \in \mathrm{H}_{k}^{*}(N)} L\left(1, \operatorname{sym}^{2} f\right)^{ \pm \ell}=\zeta(2)^{ \pm \ell}
$$


alors que le théorème $\mathrm{B}$ et le corollaire $\mathrm{B}$ impliquent

$$
\lim _{\substack{N \rightarrow \infty \\ N \in \mathcal{N}_{\text {fri }}}} \frac{1}{\zeta(2)} \sum_{f \in \mathrm{H}_{k}^{*}(N)} \omega(f) L\left(1, \operatorname{sym}^{2} f\right) L\left(1, \operatorname{sym}^{2} f\right)^{ \pm \ell}=0 .
$$

La raison est simplement que $\varphi(N) / N$ tend vers 0 lorsque $N$ tend vers $+\infty$ en restant dans $\mathcal{N}_{\text {fri }}$.

Remarque C. Si $N=p_{1} \cdots p_{j}$, alors $\log N \sim p_{j}$. Autrement dit $P^{+}(N) \leq$ $\log N$ pour $N \in \mathcal{N}_{\text {fri }}$, où $P^{+}(N)$ est le plus grand facteur premier de $N$. On peut donc voir $\log N$ comme la phase de taille des facteurs premiers de $N$ au sens suivant : si tous les facteurs premiers de $N$ sont plus grands que $\log N$, alors moyenne harmonique et moyenne naturelle sont equivalentes, par contre si tous les facteurs premiers de $N$ sont plus petits que $\log N$, alors moyenne harmonique et moyenne naturelle ne sont pas equivalentes. La situation où $N$ a des facteurs au dessus et au dessous de $\log N$ est très compliquée.

Le cas où $\ell=2$ dans le corollaire $B$ correspondant à la valeur moyenne de $L\left(1, \operatorname{sym}^{2} f\right)$, grâce à la formule (3.18) ci-dessous, a reçu une attention particulière (voir [17], [1], [23], [13]). En particulier Lau [13] a montré que si $N$ est un nombre premier, on a

$$
\sum_{f \in \mathrm{H}_{2}^{*}(N)} L\left(1, \operatorname{sym}^{2} f\right)=\frac{\pi^{4}}{432} N+O\left(N^{9 / 10}[\log (2 N)]^{c}\right),
$$

où $c>0$ est une constante absolue. Ici nous proposons un résultat plus général et plus précis. On définit la fonction $\psi$ par

$$
\psi(N):=N \prod_{p \mid N}\left(1-\frac{1}{p}\right)\left(1-\frac{1}{p^{2}}\right) .
$$

Théorème C. Soit $k>0$ un entier pair. Soit $\varepsilon>0$, alors pour tout entier $N$ sans facteur carré, on a

$$
\sum_{f \in \mathrm{H}_{k}^{*}(N)} L\left(1, \operatorname{sym}^{2} f\right)=\frac{\pi^{4}}{432}(k-1) \psi(N)+O_{\varepsilon, k}\left(N^{1 / 2+\varepsilon}\right) .
$$

En particulier si $N$ est un entier sans facteur carré vérifiant $P^{-}(N) \geq$ $N^{1 / 2-\varepsilon}$, alors

$$
\sum_{f \in \mathrm{H}_{k}^{*}(N)} L\left(1, \operatorname{sym}^{2} f\right)=\frac{\pi^{4}}{432}(k-1) N+O_{\varepsilon, k}\left(N^{1 / 2+\varepsilon}\right),
$$

où la constante impliquée dans le symbole $O$ ne dépend que de $\varepsilon$ et $k$. 


\section{Notations et boîte à outils arithmétiques}

L'objet de cette partie est d'introduire les notations et outils de la théorie analytique des nombres nécessaires à la bonne compréhension du texte.

On note $\mathbb{N}$ l'ensemble de tous les entiers positifs y compris 0 et $\mathbb{N}^{*}$ l'ensemble des entiers strictement positifs. De même $\mathbb{Z}$ et $\mathbb{Z}^{*}$ désignent respectivement l'ensemble des entiers et l'ensemble des entiers non nuls. L'ensemble des nombres premiers est noté $\mathcal{P}$.

Pour éviter la multiplication des notations, $\varepsilon>0, C>0, \xi>0$ sont des constantes absolues (c'est-à-dire ne dépendant d'aucune des variables du texte, le poids des formes modulaires étant fixé, les constantes absolues peuvent en dépendre) pouvant prendre des valeurs différentes à chacune de leurs apparitions (on évite ainsi l'introduction de $\varepsilon_{1}, \varepsilon_{2}, \ldots$ ). De même, pour chaque $\varepsilon>0$, la notation $\iota(\varepsilon)$ désigne une valeur strictement positive ne dépendant que de $\varepsilon$ (et donc d'aucune autre variable du texte). Là encore, même si $\varepsilon$ reste fixé, $\iota(\varepsilon)$ peut changer à chaque apparition.

Les vecteurs seront notés en gras. Si $\mathbf{v}$ est un vecteur à $\ell$ coordonnées, celles-ci seront désignées par $v_{1}, \ldots, v_{\ell}$. On note alors $|\mathbf{v}|:=v_{1} \cdots v_{\ell}$ et $\operatorname{tr} \mathbf{v}:=v_{1}+\cdots+v_{\ell}$

Le symbole de Kronecker est

$$
\delta(m, n):= \begin{cases}1 & \text { si } m=n \\ 0 & \text { sinon. }\end{cases}
$$

Si $n$ et $N$ sont des entiers de $\mathbb{N}^{*}$, on note $n^{(N)}$ et $n_{N}$ les uniques entiers tels que

$$
\left\{\begin{array}{l}
n=n^{(N)} n_{N} \\
n_{N} \mid N^{\infty} \\
\left(n^{(N)}, N\right)=1
\end{array}\right.
$$

Si $n$ est entier et $p$ premier, on note $v_{p}(n)$ la valuation $p$-adique de $n$. Le noyau impair de $n$ est alors

$$
N_{\mathrm{imp}}(n):=\prod_{\substack{p \in \mathcal{P} \\ v_{p}(n) \text { impair }}} p .
$$

La fonction constante égale à un est notée $\mathbb{1}$. La fonction caractéristique des entiers premiers à un entier $N$ est

$$
\mathbb{1}^{(N)}(n):= \begin{cases}1 & \text { si }(n, N)=1 \\ 0 & \text { sinon. }\end{cases}
$$


La fonction caractéristique des entiers ayant mêmes diviseurs premiers que $N$ est

$$
\mathbb{1}_{N}(n):= \begin{cases}1 & \text { si } n \mid N^{\infty} \\ 0 & \text { sinon. }\end{cases}
$$

On définit alors

$$
\mu_{N}^{+}(n):=\frac{\mathbb{1}_{N}(n)}{n} \quad \text { et } \quad \mu_{N}^{-}(n)=\frac{\mathbb{1}_{N}(n) \mu(n)}{n} .
$$

Enfin, la fonction caractéristique des carrés premiers à $N$ sera nécessaire,

$$
\square^{(N)}(n):= \begin{cases}1 & \text { si } n=d^{2} \text { avec }(d, N)=1 ; \\ 0 & \text { sinon. }\end{cases}
$$

Si $\ell \in \mathbb{N}^{*}$, on définit la fonction $\tau_{\ell}$ sur $\mathbb{N}^{*}$ par

$$
\tau_{\ell}(n):=\#\left\{\mathbf{n} \in \mathbb{N}^{\ell}:|\mathbf{n}|=n\right\} .
$$

On simplifie l'écriture en posant $\tau:=\tau_{2}$. La série de Dirichlet de la fonction multiplicative $\tau_{\ell}$ est $\zeta^{\ell}$ où $\zeta$ est la fonction zeta de Riemann définie pour Re $s>1$ par

$$
\zeta(s):=\prod_{p \in \mathcal{P}} \zeta_{p}(s)=\sum_{n \in \mathbb{N}^{*}} n^{-s} \quad \text { avec } \quad \zeta_{p}(s):=\left(1-p^{-s}\right)^{-1} .
$$

Pour tout $\ell \in \mathbb{N}^{*}$ et tout $t \geq 1$, on a

$$
\sum_{n \leq t} \tau_{\ell}(n) \leq t[\log (e t)]^{\ell-1}
$$

Cela résulte du produit de convolution $\tau_{\ell}=\tau_{\ell-1} * \mathbb{1}$. On a $\tau_{\ell}(n) \leq \tau(n)^{\ell}$ de sorte que pour tout $\varepsilon>0$, il existe une constante $\iota(\varepsilon)>0$ telle que pour tous entiers $\ell$ et $n$ on a

$$
\tau_{\ell}(n) \leq\left[\iota(\varepsilon) n^{\varepsilon}\right]^{\ell}
$$

Si $q \in \mathbb{N}^{*}$, la fonction $\zeta^{(q)}$ est la fonction zeta de Riemann privée de ses facteurs euleriens d'indices les diviseurs premiers de $q$. Ainsi, pour $\Re e s>1$,

$$
\zeta^{(q)}(s):=\prod_{\substack{p \in \mathcal{P} \\(p, q)=1}} \zeta_{p}(s)=\sum_{\substack{n \in \mathbb{N}^{*} \\(n, q)=1}} n^{-s} .
$$

Si $n \in \mathbb{N}^{*}$, la fonction $\log _{n}$ désigne l'itéré $n$ fois de la fonction log,

$$
\log _{n}:= \begin{cases}\log & \text { si } n=1 \\ \log \circ \log _{n-1} & \text { si } n>1\end{cases}
$$

Si $s \in \mathbb{C}$, on désignera sa partie réelle par $\sigma$ et sa partie imaginaire par $\tau$. Enfin, la droite $\Re e s=r$ est notée $(r)$. 


\section{Cadre modulaire}

\subsection{Formes primitives}

Pour tout entier $N \geq 1$, on note

$$
\Gamma_{0}(N):=\left\{\left(\begin{array}{ll}
a & b \\
c & d
\end{array}\right):(a, b, c, d) \in \mathbb{Z}^{4}, a d-b c=1, N \mid c\right\} .
$$

Pour tout entier $k \geq 2$ pair, on appelle forme parabolique de poids $k$ et de niveau $N$ toute fonction $f$, holomorphe sur le demi-plan de Poincaré $\mathcal{H}:=\{z \in \mathbb{C}: \Im m z>0\}$, vérifiant

- pour tout $z \in \mathcal{H}$ et toute matrice $\left(\begin{array}{ll}a & b \\ c & d\end{array}\right) \in \Gamma_{0}(N)$,

$$
f\left(\frac{a z+b}{c z+d}\right)=(c z+d)^{k} f(z)
$$

- la fonction

$$
z \mapsto(\Im m z)^{k / 2}|f(z)|
$$

est bornée sur $\mathcal{H}$.

Remarque 1. Dans cette définition, «le» niveau n'est pas défini de manière unique : par exemple, toute forme de niveau $N$ est aussi de niveau $2 N$ puisque $\Gamma_{0}(2 N) \subset \Gamma_{0}(N)$. Rigoureusement, il faut donc avoir à l'esprit qu'une forme parabolique de niveau $N$ est un couple $(f, N)$.

L'ensemble $S_{k}(N)$ de ces formes est un espace hermitien lorsqu'on le munit du produit scalaire

$$
\langle f, g\rangle:=\int_{D_{0}(N)} f(z) \overline{g(z)} y^{k} \frac{\mathrm{d} x \mathrm{~d} y}{y^{2}}
$$

avec $D_{0}(N)$ un domaine fondamental du quotient à gauche de $\mathcal{H} \cup \mathbb{Q} \cup\{\infty\}$ par $\Gamma_{0}(N)$ pour l'action homographique [Iwa97, $\S 2.7$ et 3.3].

Si $f \in S_{k}(N)$, elle admet un développement de Fourier qu'on écrit

$$
f(z)=\sum_{n=1}^{+\infty} \widehat{f}(n) \exp (2 \pi i n z) .
$$

Ayant fixé une base orthogonale $\mathscr{B}_{k}(N)$ de $S_{k}(N)$ on a la formule de trace de Petersson, démontrée par exemple dans [8, corollaire 2.2], et rappelée dans le 
Lemme 2 (Formule de trace de Petersson). Soit $k \geq 2$ un entier pair; soient $N \geq 1, m \geq 1$ et $n \geq 1$ des entiers. Alors

$$
\begin{aligned}
& \sum_{f \in \mathscr{B}_{k}(N)} \frac{\Gamma(k-1)}{(4 \pi)^{k-1}\langle f, f\rangle} \frac{\widehat{f}(m) \widehat{f}(n)}{(m n)^{(k-1) / 2}}=\delta(m, n) \\
& \quad+O\left(k^{-5 / 6} \frac{\tau(N)}{N} \frac{(m, n, N) \tau_{3}[(m, n)]}{\sqrt{(m, N)+(n, N)}} \sqrt{\frac{m n}{\sqrt{m n}+k N}} \log (2 m n N)\right) .
\end{aligned}
$$

Pour simplifier l'écriture, on notera

$$
\omega(f):=\frac{\Gamma(k-1)}{(4 \pi)^{k-1}\langle f, f\rangle} .
$$

Lorsque $d$ et $N^{\prime}$ sont deux diviseurs de $N$ tels que $N^{\prime}<N$ et $d \mid \frac{N}{N^{\prime}}$ et si $f \in S_{k}\left(N^{\prime}\right)$ alors $z \mapsto f(d z)$ est une forme de $S_{k}(N)$. L'espace engendré par de telles formes est appelé espace des formes anciennes. Son orthogonal pour le produit (3.1) est l'espace des formes nouvelles. Une raison pour laquelle on fait une distinction entre formes anciennes et nouvelles est l'existence d'une base orthogonale privilégiée de l'espace des formes nouvelles que l'on décrit maintenant.

Pour tout $n \in \mathbb{N}^{*}$, on définit le $n^{\mathrm{e}}$ opérateur de Hecke [10, chapitre 6] par

$$
\begin{aligned}
T_{n}: S_{k}(N) & \rightarrow S_{k}(N) \\
f & \mapsto\left[z \mapsto \frac{1}{n} \sum_{a d=n} \mathbb{1}^{(N)}(a) a^{k} \sum_{0 \leq b<d} f\left(\frac{a z+b}{c z+d}\right)\right] .
\end{aligned}
$$

Une base orthogonale privilégiée de l'espace des formes nouvelles est la base des formes primitives. Les formes primitives vérifient les propriétés suivantes.

- Le développement de Fourier s'écrit

$$
f(z)=\sum_{n=1}^{+\infty} \lambda_{f}(n) n^{(k-1) / 2} \exp (2 \pi i n z) .
$$

- On a la normalisation $\lambda_{f}(1)=1$.

- Pour tout entier $n \geq 1$

$$
T_{n} f=\lambda_{f}(n) n^{(k-1) / 2} f .
$$

- Pour tous entiers $m \geq 1$ et $n \geq 1$,

$$
\lambda_{f}(m) \lambda_{f}(n)=\sum_{d \mid(m, n)} \mathbb{1}^{(N)}(d) \lambda_{f}\left(\frac{m n}{d^{2}}\right) .
$$

- Pour tout entier $n$, on a $\lambda_{f}(n) \in \mathbb{R}$. 
On note $\mathrm{H}_{k}^{*}(N)$ l'ensemble des formes primitives de poids $k$ et de niveau $N$. Lorsque $N$ est sans facteur carré, on a l'estimation (voir [8, Corollary 2.14])

$$
\mathrm{H}_{k}^{*}(N)=\frac{k-1}{12} \varphi(N)+O\left((k N)^{2 / 3}\right)
$$

Grâce, entre autres, aux travaux de Deligne, il existe, pour tout nombre premier $p$, des nombres complexes $\alpha_{f}(p)$ et $\beta_{f}(p)$ tels que, pour tout entier $\nu \geq 0$ le réel $\lambda_{f}\left(p^{\nu}\right)$ s'écrit

$$
\lambda_{f}\left(p^{\nu}\right)=\frac{\alpha_{f}(p)^{\nu+1}-\beta_{f}(p)^{\nu+1}}{\alpha_{f}(p)-\beta_{f}(p)} .
$$

Les complexes $\alpha_{f}(p)$ et $\beta_{f}(p)$ vérifient

$$
\begin{cases}\alpha_{f}(p)=\beta_{f}(p)=0 & \text { si } p^{2} \mid N \\ \alpha_{f}(p)=\varepsilon_{f}(p) p^{-1 / 2}, \beta_{f}(p)=0 & \text { si } p \| N ; \\ \left|\alpha_{f}(p)\right|=1, \beta_{f}(p)=\alpha_{f}(p)^{-1} & \text { si }(p, N)=1\end{cases}
$$

où $\varepsilon_{f}(p)= \pm 1$. On étend la définition de $\varepsilon_{f}$ aux entiers sans facteur carré par multiplicativité. Une conséquence de (3.6) et de (3.3) est la majoration, valable pour tout $n$ entier,

$$
\left|\lambda_{f}(n)\right| \leq \tau(n)
$$

Une autre conséquence de (3.6) est que lorsque $N$ est sans facteur carré, on peut récrire (3.3) en

$$
\lambda_{f}(m) \lambda_{f}(n)=\frac{\varepsilon_{f}\left[N_{\mathrm{imp}}\left(m_{N} n_{N}\right)\right]}{\sqrt{m_{N} n_{N}}} \sum_{d \mid\left(m^{(N)}, n^{(N)}\right)} \lambda_{f}\left(\frac{m^{(N)} n^{(N)}}{d^{2}}\right) .
$$

Remarque 3. Soit $\mathcal{K}=\{2,4,6,8,10,14\}$. Il n'existe pas de formes paraboliques de poids $k \in \mathcal{K}$ et de niveau 1 [20, §3.2]. Cela implique que, pour $k \in \mathcal{K}$ et $N \in \mathcal{P}, \mathrm{H}_{k}^{*}(N)$ est une base orthogonale de tout l'espace $S_{k}(N)$. Il est crucial de garder en tête que ce n'est pas le cas lorsque $N$ n'est pas premier ou lorsque $k$ n'est pas dans $\mathcal{K}$.

Dans [8, corollaire 2.10], lorsque $N$ est sans facteur carré, Iwaniec, Luo et Sarnak ont établi une formule de trace semblable à celle du lemme 2 mais avec une sommation sur les seules formes primitives. On rappelle le résultat dans le 
Lemme 4 (Formule de trace d'Iwaniec, Luo \& Sarnak) Soient $N \geq 1$ un entier sans facteur carré et $m \geq 1, n \geq 1$ des entiers tels que $(m, N)=1$ et $\left(n, N^{2}\right) \mid N$. Alors

$$
\begin{aligned}
\sum_{f \in \mathrm{H}_{k}^{*}(N)} \omega(f) \lambda_{f}(m) & \lambda_{f}(n)=\frac{\varphi(N)}{N} \delta(m, n) \\
+ & O\left(k^{-5 / 6} \frac{\tau(N)^{2}}{N} \frac{(m n)^{1 / 4} \tau_{3}[(m, n)]}{\sqrt{(n, N)}} \log (2 m n N)\right) .
\end{aligned}
$$

En particulier, le choix de $m=n=1$ dans le lemme 4 conduit à

$$
\sum_{f \in \mathrm{H}_{k}^{*}(N)} \omega(f)=\frac{\varphi(N)}{N}\left\{1+O\left(k^{-5 / 6} \frac{\tau(N)^{2}}{\varphi(N)} \log (2 N)\right)\right\} .
$$

Lorsque $k \in \mathcal{K}$ et $N \in \mathcal{P}$, la remarque 3 et le lemme 2 impliquent le résultat plus précis $($ en $N)$ du

Lemme 5. Soient $k \in\{2,4,6,8,10,14\}, N \in \mathcal{P}$ et $m \geq 1, n \geq 1$ des entiers tels que $(m, N)=1$ et $\left(n, N^{2}\right) \mid N$. Alors

$$
\begin{aligned}
\sum_{f \in \mathrm{H}_{k}^{*}(N)} \omega(f) \lambda_{f}(m) & \lambda_{f}(n)=\delta(m, n) \\
+ & O\left(k^{-4 / 3} \frac{1}{N^{3 / 2}} \frac{(m n)^{1 / 2} \tau_{3}[(m, n)]}{\sqrt{(n, N)}} \log (2 m n N)\right) .
\end{aligned}
$$

\subsection{Fonctions $L$}

Soient $r \geq 1$ un entier et, pour tout premier $p$, une matrice diagonale, $\varpi_{p}=\operatorname{diag}\left[\alpha_{1}(p), \ldots, \alpha_{r}(p)\right]$ à coefficients complexes de norme inférieure ou égale à 1 et égale à 1 sauf pour un nombre fini de premiers. On associe à cette matrice la fonction $L$ «locale»

$$
L\left(s, \varpi_{p}\right):=\prod_{i=1}^{r}\left[1-\alpha_{i}(p) p^{-s}\right]^{-1} .
$$

On construit alors la fonction $L$ 《globale»

$$
L(s, \varpi)=\prod_{p \in \mathcal{P}} L\left(s, \varpi_{p}\right) .
$$

Le produit (3.10) est convergent pour $\sigma>1$ et se développe en la série de Dirichlet

$$
L(s, \varpi):=\sum_{n=1}^{+\infty} \frac{\lambda_{\varpi}(n)}{n^{s}}
$$


On fait l'hypothèse $\mathfrak{E}$ : on suppose qu'il existe des complexes $\mu_{\varpi, i}$ de parties réelles strictement supérieures à $-\frac{1}{2}$ tels que la fonction

$$
\Lambda(s, \varpi)=L\left(s, \varpi_{\infty}\right) L(s, \varpi)
$$

avec

$$
L\left(s, \varpi_{\infty}\right)=\pi^{-s r / 2} \prod_{i=1}^{r} \Gamma\left(\frac{s+\mu_{\varpi, i}}{2}\right)
$$

est entière et satisfait l'équation fonctionnelle

$$
q_{\varpi}^{s / 2} \Lambda(s, \varpi)=\varepsilon_{\varpi} q_{\varpi}^{(1-s) / 2} \overline{\Lambda(1-\bar{s}, \varpi)} .
$$

Dans (3.12), la paramètre $q_{\varpi}$ est un entier appelé conducteur de $\varpi$ et $\varepsilon_{\varpi}$ est un complexe de norme 1 (mal) nommé signe de l'équation fonctionnelle. Sous cette hypothèse, on a (voir, par exemple [16]) la borne de convexité : pour tout $\varepsilon>0$, il existe $C>0$ (ne dépendant que de $r$ et $\varepsilon$ ) tel que

$$
|L(s, \varpi)| \leq C\left[q_{\varpi} \prod_{i=1}^{r}\left|\frac{1}{2}+i \tau-\mu_{\varpi, i}\right|\right]^{\max \{(1-\sigma) / 2,0\}+\varepsilon} \quad(\sigma \geq 0) .
$$

Soit $f \in \mathrm{H}_{k}^{*}(N)$. Si on choisit, pour $\varpi_{p}$, la matrice $f_{p}=\operatorname{diag}\left[\alpha_{f}(p), \beta_{f}(p)\right]$ on obtient la fonction $L$ de $f$. L'hypothèse $\mathfrak{E}$ est due à Hecke. Les paramètres associés sont

$$
\left\{\begin{aligned}
r & =2 & & \\
\alpha_{1}(p) & =\alpha_{f}(p) & \alpha_{2}(p) & =\beta_{f}(p) \\
\mu_{f, 1} & =\frac{k-1}{2} & & \mu_{f, 2}=\frac{k+1}{2} \\
q_{f} & =N . & &
\end{aligned}\right.
$$

On suppose $N$ sans facteur carré. Si on choisit, pour $\varpi_{p}$, le carré symétrique $\operatorname{sym}^{2} f_{p}=\operatorname{diag}\left[\alpha_{f}(p)^{2}, \alpha_{f}(p) \beta_{f}(p), \beta_{f}(p)^{2}\right]$ de $f_{p}$, on obtient la fonction $L$ de carré symétrique de $f$. L'hypothèse $\mathfrak{E}$ est due à Shimura et Li. Les paramètres associés sont

$$
\left\{\begin{array}{rlrl}
r & =3 & & \\
\alpha_{1}(p) & =\alpha_{f}(p)^{2} \quad \alpha_{2}(p)=\alpha_{f}(p) \beta_{f}(p) & \alpha_{3}(p)=\beta_{f}(p)^{2} \\
\mu_{\mathrm{sym}^{2} f, 1} & =1 & \mu_{\mathrm{sym}^{2} f, 2}=k-1 & \mu_{\mathrm{sym}^{2} f, 3}=k \\
q_{\mathrm{sym}^{2} f} & =N^{2} \\
\varepsilon_{\mathrm{sym}^{2} f} & =1 .
\end{array}\right.
$$

Grâce à (3.5) et (3.8) on a

$$
\rho_{f}(n):=\lambda_{\mathrm{sym}^{2} f}(n)=\sum_{\substack{a, b, c \in \mathbb{N} \\ a^{2} b c=n}} \mathbb{1}^{(N)}(a b) \frac{\mathbb{1}_{N}(c)}{c} \lambda_{f}\left(b^{2}\right) .
$$


Dans les deux cas précédents, la série de Dirichlet (3.11) est à coefficients réels et dans (3.12), on a alors $\overline{\Lambda(1-\bar{s}, \varpi)}=\Lambda(1-s, \varpi)$.

On aura besoin de contrôler les zéros de $L\left(s, \operatorname{sym}^{2} f\right)$ au voisinage de $s=1$. Pour cela, on utilise un résultat de Kowalski \& Michel [12, théorème 2]. Grâce à un résultat de Ramakrishnan [2, corollaire du théorème $\mathrm{A}]$, on a, puisque $N$ est sans facteur carré,

$$
\left\{\operatorname{sym}^{2} f: f \in \mathrm{H}_{k}^{*}(N)\right\} \simeq \mathrm{H}_{k}^{*}(N) .
$$

En particulier, si $A$ est une fonction de $\left\{\operatorname{sym}^{2} f: f \in \mathrm{H}_{k}^{*}(N)\right\}$ dans $\mathbb{C}$, alors

$$
\sum_{g \in\left\{\operatorname{sym}^{2} f: f \in \mathrm{H}_{k}^{*}(N)\right\}} A(g)=\sum_{f \in \mathrm{H}_{k}^{*}(N)} A\left(\operatorname{sym}^{2} f\right)
$$

et un cas particulier du résultat de Kowalski \& Michel s'énonce

Lemme 6 (Kowalski \& Michel). Soit $k>0$ un entier pair. Soient $\alpha \geq \frac{3}{4}$, $T \geq 2, N$ un entier sans facteur carré et $f \in \mathrm{H}_{k}^{*}(N)$. On définit

$$
N\left(\operatorname{sym}^{2} f ; \alpha, T\right)=\#\left\{\rho \in \mathbb{C}: \Re e \rho \geq \alpha,|\Im m \rho| \leq T, L\left(\rho, \operatorname{sym}^{2} f\right)=0\right\} .
$$

Alors il existe une constante absolue $\xi>0$ ne dépendant que de $k$ telle que

$$
\sum_{f \in \mathrm{H}_{k}^{*}(N)} N\left(\operatorname{sym}^{2} f ; \alpha, T\right) \leq \xi T^{\xi} N^{17(1-\alpha) /(2 \alpha-1)} .
$$

Ce lemme permet d'établir une «quasi hypothèse de Riemann»: si $\eta$ est un réel positif assez petit, disons $0<\eta \leq \frac{1}{22}$, on définit

$$
\mathrm{H}_{k}^{+}(N ; \eta)=\left\{f \in \mathrm{H}_{k}^{*}(N): L\left(s, \operatorname{sym}^{2} f\right) \neq 0, \sigma \geq 1-4 \eta,|\tau| \leq 2[\log (2 N)]^{3}\right\}
$$

et

$$
\mathrm{H}_{k}^{-}(N ; \eta)=\mathrm{H}_{k}^{*}(N) \backslash \mathrm{H}_{k}^{+}(N ; \eta) .
$$

Alors il existe $\xi>0$ telle que, pour tout $\left.\eta \in] 0, \frac{1}{22}\right]$ on a

$$
\# \mathrm{H}_{k}^{-}(N ; \eta) \leq \xi N^{19 \eta}[\log (2 N)]^{\xi} .
$$

On aura besoin de majorer $\omega(f)$ pour $f \in \mathrm{H}_{k}^{*}(N)$. Grâce à l'égalité

$$
\omega(f)=\frac{2 \pi^{2}}{(k-1) N L\left(1, \operatorname{sym}^{2} f\right)}
$$

(dont une preuve se déduit, par exemple, du lemme 2.5 de [8]) et à la majoration (1.1), on a

$$
\omega(f) \ll_{k} \frac{\log (2 N)}{N} .
$$


Les puissances de $L\left(s, \operatorname{sym}^{2} f\right)$ admettent un développement en série de Dirichlet pour $\sigma>1$. Pour le donner, il est nécessaire d'introduire, pour tout entier $\ell \geq 1$ et tout $\mathbf{b} \in \mathbb{N}^{\ell}$, l'ensemble

$$
\mathcal{E}_{\ell}(\mathbf{b})=\left\{\mathbf{d} \in \mathbb{N}^{\ell-1}: d_{j} \mid\left(\frac{b_{1} \cdots b_{j}}{d_{1} \cdots d_{j-1}}, b_{j+1}\right)^{2} \quad(1 \leq j \leq \ell-1)\right\}
$$

avec les conventions suivantes : $\mathbb{N}^{0}=\{1\}, \mathcal{E}_{1}(\mathbf{b})=\{1\}$ et $d_{1} \cdots d_{0}=1$. On a alors, pour tout entier $\ell \geq 1$ et tout $\sigma>1$,

$$
L\left(s, \operatorname{sym}^{2} f\right)^{ \pm \ell}=\sum_{n=1}^{+\infty} \rho_{f}^{( \pm \ell)}(n) n^{-s}
$$

où $\rho_{f}^{( \pm \ell)}$ est la fonction multiplicative donnée grâce à (3.16) par

$$
\rho_{f}^{(+\ell)}(n)=\sum_{\substack{\mathbf{a}, \mathbf{b}, \mathbf{c} \in \mathbb{N}^{\ell} \\\left|\mathbf{a}^{2} \mathbf{b} \mathbf{c}\right|=n}} \mathbb{1}^{(N)}(|\mathbf{a b}|) \frac{\mathbb{1}_{N}(|\mathbf{c}|)}{|\mathbf{c}|} \sum_{\mathbf{d} \in \mathcal{E}_{\ell}(\mathbf{b})} \lambda_{f}\left[\left(\frac{|\mathbf{b}|}{|\mathbf{d}|}\right)^{2}\right]
$$

et, grâce à (3.8) par

$$
\begin{aligned}
\rho_{f}^{(-\ell)}(n)= & \sum_{\substack{\mathbf{a}, \mathbf{b}, \mathbf{c}, \mathbf{e} \in \mathbb{N}^{\ell} \\
\left|\mathbf{a b}^{2} \mathbf{c}^{\mathbf{3}} \mathbf{e}\right|=n}} \mathbb{1}^{(N)}(|\mathbf{a b c}|) \frac{\mathbb{1}_{N}(|\mathbf{e}|)}{|\mathbf{e}|} \times \\
& {\left[\prod_{1 \leq j \leq \ell} \mu\left(a_{j} b_{j} c_{j}\right) \mu\left(b_{j}\right) \mu\left(e_{j}\right)\right] \sum_{\mathbf{d} \in \mathcal{E}_{\ell}(\mathbf{a b})} \lambda_{f}\left[\left(\frac{|\mathbf{a b}|}{|\mathbf{d}|}\right)^{2}\right] . }
\end{aligned}
$$

Les définitions (3.20) et (3.21) avec la majoration de Deligne (3.7) impliquent pour tout $\varepsilon>0$ l'existence d'une constante $\iota(\varepsilon)>0$ ne dépendant que de $\varepsilon$ telle que pour tout $\sigma>1+\varepsilon$ et $\ell \in \mathbb{N}^{*}$, on a la majoration

$$
\left|L\left(s, \operatorname{sym}^{2} f\right)^{ \pm \ell}\right| \leq[\iota(\varepsilon) \zeta(\sigma-\varepsilon)]^{\ell} .
$$

Enfin, on utilisera une majoration en moyenne plus puissante (en le paramètre $N$ ) que la borne de convexité (3.13) et due à Iwaniec et Michel [9].

Lemme 7 (Iwaniec \& Michel). Soit $k>0$ un entier pair. Pour tout $\varepsilon>0$, il existe un réel $C>0$ tel que pour tout entier $N$ sans facteur carré et tout complexe $s$ de partie réelle $\frac{1}{2}$, on a

$$
\sum_{f \in \mathrm{H}_{k}^{*}(N)}\left|L\left(s, \operatorname{sym}^{2} f\right)\right|^{2} \leq C|s|^{8} N^{1+\varepsilon} .
$$

Remarque 8. Dans tout cet article, le poids des formes modulaires est fixé. Ainsi, les termes d'erreur et les constantes mêmes qualifiés d'absolues peuvent en dépendre. 


\section{Calcul effectif des moments et preuve du théorème B}

\subsection{Lemmes de Luo effectifs}

Dans le but d'obtenir une estimation d'ordre $\ell$ de la valeur $L\left(1, \operatorname{sym}^{2} f\right)$ avec terme d'erreur faisant apparaître la dépendance en $\ell$, il est nécessaire de préciser les termes d'erreurs dans des estimations dues à Luo. C'est l'objet de cette partie.

On précise le lemme 2 de [14].

Lemme 9. Il existe un réel $\xi>0$ tel que, pour tout entier $N$ sans facteur carré, toute forme primitive $f \in \mathrm{H}_{k}^{*}(N)$ et tout réel $\left.\left.\eta \in\right] 0, \frac{1}{22}\right]$, si $L\left(s, \operatorname{sym}^{2} f\right)$ ne s'annule pas dans le domaine

$$
1-4 \eta \leq \sigma<1 \quad \text { et } \quad|\tau| \leq 2[\log (2 N)]^{3}
$$

alors pour tout $s \in \mathbb{C}$ tel que

$$
1-\eta \leq \sigma<1 \quad \text { et } \quad|\tau| \leq[\log (2 N)]^{2}
$$

on $a$

$$
\left|L\left(s, \operatorname{sym}^{2} f\right)\right| \leq \exp \left\{D_{+}(\eta)[\log (2 N)]^{\theta(\eta)}\right\}
$$

avec

$$
\theta(\eta):=\frac{\log [(1+\eta) /(1-2 \eta)]}{\log [(1+5 \eta) /(1-2 \eta)]} \quad \text { et } \quad D_{+}(\eta):=\frac{\xi}{\eta} \log \left(\frac{\xi}{\eta}\right)
$$

Démonstration. Sur le domaine

$$
D(\eta, N):=\left\{s \in \mathbb{C}: 1-4 \eta \leq \sigma<1,|\tau| \leq 2[\log (2 N)]^{3}\right\} \cup\{s \in \mathbb{C}: \sigma \geq 1\},
$$

on définit

$$
F(s):=\log L\left(s, \operatorname{sym}^{2} f\right) .
$$

Grâce à (3.7), si $\sigma \geq 4$, on a

$$
\left|L\left(s, \operatorname{sym}^{2} f\right)\right| \leq\left(\sum_{n=1}^{+\infty} \frac{1}{n^{8}}\right)\left(\sum_{n=1}^{+\infty} \frac{\tau\left(n^{2}\right)}{n^{4}}\right)=: C_{1} .
$$

On déduit de (4.1) l'existence d'une constante absolue $C_{2}>0$ telle que pour $\sigma \geq 4$ on a

$$
|F(s)|^{2}=|\log | L\left(s, \operatorname{sym}^{2} f\right)||^{2}+\left|\arg L\left(s, \operatorname{sym}^{2} f\right)\right|^{2} \leq C_{2}^{2} .
$$


D'autre part, il existe une contante absolue $C_{3}>0$ telle pour $\sigma \geq 1+\eta$, on a

$$
\left|L\left(s, \operatorname{sym}^{2} f\right)\right| \leq\left(\sum_{n=1}^{+\infty} \frac{1}{n^{2}}\right)\left(\sum_{n=1}^{+\infty} \frac{\tau\left(n^{2}\right)}{n^{1+\eta}}\right) \leq\left(\frac{C_{3}}{\eta}\right)^{3} .
$$

On déduit de (4.3) que, pour $\sigma \geq 1+\eta$, on a

$$
\Re e F(s)=\log \left|L\left(s, \operatorname{sym}^{2} f\right)\right| \leq 3 \log \frac{C_{3}}{\eta} .
$$

On applique alors le théorème de Borel-Carathéodory [21] à $F$. Avec $s^{\prime}=4+i \tau, R^{\prime}=3-\eta$ et $r^{\prime}=3-2 \eta$, les équations (4.2) et (4.4) conduisent à

$$
\begin{aligned}
\max _{\left|s-s^{\prime}\right|=r^{\prime}}|F(s)| & \leq \frac{2 r^{\prime}}{R^{\prime}-r^{\prime}} \max _{\left|s-s^{\prime}\right|=R^{\prime}} \Re e F(s)+\frac{R^{\prime}+r^{\prime}}{R^{\prime}-r^{\prime}}\left|F\left(s^{\prime}\right)\right| \\
& \leq 3 \frac{14-4 \eta}{\eta} \log \frac{C_{3}}{\eta}+\frac{6-3 \eta}{\eta} C_{2} \\
& \leq \frac{C}{\eta} \log \frac{C}{\eta} .
\end{aligned}
$$

En particulier pour $s=\sigma+i \tau$ avec $1+2 \eta \leq \sigma \leq 7-2 \eta$ et $\tau \in \mathbb{R}$, on a

$$
|F(s)| \leq \frac{C}{\eta} \log \frac{C}{\eta} .
$$

Il résulte de la borne de convexité (3.13) que

$$
\Re e F(s) \leq \xi \log (2 N)
$$

pour $\frac{1}{2} \leq \sigma \leq 8$ et $|\tau| \leq[\log (2 N)]^{3}$.

On applique le théorème de Borel-Carathéodory à $F(s)$. Avec $s^{\prime \prime}:=4+i \tau$ (où $|\tau| \leq[\log (2 N)]^{3}$ ), $R^{\prime \prime}:=3+6 \eta$ et $r^{\prime \prime}:=3+5 \eta$, on déduit de (4.6) et (4.2) que

$$
\begin{aligned}
\max _{\left|s-s^{\prime \prime}\right|=r^{\prime \prime}}|F(s)| & \leq \frac{2 r^{\prime \prime}}{R^{\prime \prime}-r^{\prime \prime}} \max _{\left|s-s^{\prime \prime}\right|=R^{\prime \prime}} \Re e F(s)+\frac{R^{\prime \prime}+r^{\prime \prime}}{R^{\prime \prime}-r^{\prime \prime}}\left|F\left(s^{\prime \prime}\right)\right| \\
& \leq \xi \frac{6+10 \eta}{\eta} \log (2 N)+\frac{6+11 \eta}{\eta} C_{2} \\
& \leq \frac{\xi}{\eta} \log (2 N) .
\end{aligned}
$$

En particulier pour $s=\sigma+i \tau$ avec $1-5 \eta \leq \sigma \leq 7+5 \eta$ et $|\tau| \leq[\log (2 N)]^{3}$, on a

$$
|F(s)| \leq \frac{\xi}{\eta} \log (2 N)
$$


Maintenant on applique le théorème des trois cercles de Hadamard à $F(s)$ en choisissant trois cercles de même centre $s_{0}=2+i \tau$ (où $|\tau| \leq(\log N)^{2}$ ) et de rayons $r_{1}:=1-2 \eta, r_{2}:=2-\sigma$ (où $1-\eta \leq \sigma<1$ ), $r_{3}:=1+5 \eta$. En posant $M(r):=\max _{\left|s-s_{0}\right|=r}|F(s)|$, on a

$$
M\left(r_{2}\right) \leq M\left(r_{1}\right)^{1-a} M\left(r_{3}\right)^{a}
$$

où $a:=\log \left(r_{2} / r_{1}\right) / \log \left(r_{3} / r_{1}\right)$. En majorant $M\left(r_{1}\right)$ et $M\left(r_{3}\right)$ par $(4.5)$ et $(4.7)$ respectivement, on obtient

$$
M\left(r_{2}\right) \leq\left(\frac{C}{\eta} \log \frac{C}{\eta}\right)^{1-a}\left[\frac{\xi}{\eta} \log (2 N)\right]^{a},
$$

qui implique le résultat souhaité puisque $0<a \leq \theta(\eta)$.

Pour prouver un résultat équivalent pour la fonction $L\left(s, \operatorname{sym}^{2} f\right)^{-1}$ on a besoin d'un résultat préliminaire.

Lemme 10. Il existe un réel $\xi>0$ tel que, pour tout entier $N$ sans facteur carré, toute forme primitive $f \in \mathrm{H}_{k}^{*}(N)$ et tout réel $\left.\left.\eta \in\right] 0, \frac{1}{22}\right]$, si $L\left(s, \operatorname{sym}^{2} f\right)$ ne s'annule pas dans le domaine

$$
1-4 \eta \leq \sigma<1 \quad \text { et } \quad|\tau| \leq 2[\log (2 N)]^{3}
$$

alors pour tout $s \in \mathbb{C}$ tel que

$$
1-\eta \leq \sigma<1 \quad \text { et } \quad|\tau| \leq \frac{3}{2}[\log (2 N)]^{3}
$$

on $a$

$$
\left.|\log | L\left(s, \operatorname{sym}^{2} f\right)\right|^{-1} \mid \leq \frac{\xi}{\eta}\left[\frac{1}{\eta}+\log (2 N)\right] .
$$

Démonstration. On pose $s_{0}:=1+2 \eta+i \tau_{0}$ avec $\left|\tau_{0}\right| \leq \frac{3}{2}[\log (2 N)]^{3}$, $r:=13 \eta$ et $r^{\prime}:=3 \eta$. On va appliquer le lemme $\gamma$ de $[22]$ pour établir le résultat souhaité.

Pour $\sigma \geq 1+\eta$, on a grâce à (3.10) et (3.6) la minoration

$$
\begin{aligned}
\left|L\left(s, \operatorname{sym}^{2} f\right)\right| & \geq \prod_{p \mid N}\left(1+p^{-2-\eta}\right)^{-1} \prod_{p \nmid N}\left(1+p^{-1-\eta}\right)^{-3} \\
& \geq C \prod_{p}\left(1+p^{-1-\eta}\right)^{-3} \geq e^{-C / \eta} .
\end{aligned}
$$

En utilisant cette inégalité avec $s=s_{0}$ et (3.13) avec le choix $\varepsilon=1$, on déduit

$$
\left|\frac{L\left(s, \operatorname{sym}^{2} f\right)}{L\left(s_{0}, \operatorname{sym}^{2} f\right)}\right| \leq e^{\xi[1 / \eta+\log (2 N)]}
$$

pour $\sigma \geq 1-11 \eta$ et $|\tau| \leq 2[\log (2 N)]^{3}$. En particulier l'inégalité précédente est vraie pour $\left|s-s_{0}\right| \leq r$. 
En prenant la dérivée logarithmique de deux côtés de (3.10), on peut écrire, pour $\sigma>1$,

$$
\begin{aligned}
& \frac{L^{\prime}\left(s, \operatorname{sym}^{2} f\right)}{L\left(s, \operatorname{sym}^{2} f\right)}= \\
& \quad=-\sum_{p \mid N} \frac{\log p}{p^{s+1}-1}-\sum_{p \nmid N}\left[\frac{\alpha_{f}(p)^{2} \log p}{p^{s}-\alpha_{f}(p)^{2}}+\frac{\log p}{\alpha_{f}(p)^{2} p^{s}-1}+\frac{\log p}{p^{s}-1}\right] .
\end{aligned}
$$

Grâce à (3.6), on déduit de (4.9) que

$$
\left|\frac{L^{\prime}\left(s_{0}, \operatorname{sym}^{2} f\right)}{L\left(s_{0}, \operatorname{sym}^{2} f\right)}\right| \leq \sum_{p} \frac{4 \log p}{p^{1+\eta}-1} \leq \frac{C}{\eta} \leq \frac{\xi}{\eta}\left[\frac{1}{\eta}+\log (2 N)\right] .
$$

D'après l'hypothèse, $L\left(s, \operatorname{sym}^{2} f\right) \neq 0$ dans la partie $\sigma \geq 1+2 \eta-2 r^{\prime}$ du cercle $\left|s-s_{0}\right| \leq r$. Ainsi la fonction $L\left(s, \operatorname{sym}^{2} f\right)$ vérifie toutes les conditions du lemme $\gamma$ avec les paramètres

$$
\begin{array}{rlrl}
s_{0}:=1+2 \eta+i \tau_{0}, & \left|\tau_{0}\right| & \leq \frac{3}{2}[\log (2 N)]^{3}, & r:=13 \eta, \\
r^{\prime}:=3 \eta \quad \text { et } & M:=\xi\left[\frac{1}{\eta}+\log (2 N)\right] . &
\end{array}
$$

Ce lemme fournit alors l'estimation suivante :

$$
\left|\frac{L^{\prime}\left(s, \operatorname{sym}^{2} f\right)}{L\left(s, \operatorname{sym}^{2} f\right)}\right| \leq \frac{\xi}{\eta}\left[\frac{1}{\eta}+\log (2 N)\right] \quad\left(\left|s-s_{0}\right| \leq r^{\prime}\right) .
$$

En particulier pour $\sigma \in[1-\eta, 1+5 \eta]$ et $|\tau| \leq \frac{3}{2}[\log (2 N)]^{3}$, on a

$$
\left|\frac{L^{\prime}\left(s, \operatorname{sym}^{2} f\right)}{L\left(s, \operatorname{sym}^{2} f\right)}\right| \leq \frac{\xi}{\eta}\left[\frac{1}{\eta}+\log (2 N)\right] .
$$

Puisque

$$
\begin{aligned}
& \log \left|L\left(s, \operatorname{sym}^{2} f\right)\right|^{-1}= \\
& \quad \Re e \log L\left(1+2 \eta+i \tau, \operatorname{sym}^{2} f\right)^{-1}+\int_{\sigma}^{1+2 \eta} \Re e\left[\frac{L^{\prime}\left(u+i \tau, \operatorname{sym}^{2} f\right)}{L\left(u+i \tau, \operatorname{sym}^{2} f\right)}\right] \mathrm{d} u
\end{aligned}
$$

la borne (3.13) avec le choix $\varepsilon=1$ et (4.10) donnent

$$
\left.|\log | L\left(s, \operatorname{sym}^{2} f\right)\right|^{-1} \mid \leq \xi \log (2 N)+\frac{\xi}{\eta}\left[\frac{1}{\eta}+\log (2 N)\right]
$$

pour

$$
\sigma \geq 1-\eta \quad \text { et } \quad|\tau| \leq \frac{3}{2}[\log (2 N)]^{3} .
$$

Cela achève la démonstration. 
On peut alors préciser le lemme 8 de [15] dans le

Lemme 11. Il existe un réel $\xi>0$ tel que, pour tout entier $N$ sans facteur carré, toute forme primitive $f \in \mathrm{H}_{k}^{*}(N)$ et tout réel $\left.\left.\eta \in\right] 0, \frac{1}{22}\right]$, si $L\left(s, \operatorname{sym}^{2} f\right)$ ne s'annule pas dans le domaine

$$
1-4 \eta \leq \sigma<1, \quad \text { et } \quad|\tau| \leq 2[\log (2 N)]^{3}
$$

alors pour tout $s \in \mathbb{C}$ tel que

$$
1-\eta \leq \sigma<1 \quad \text { et } \quad|\tau| \leq[\log (2 N)]^{2}
$$

on $a$

$$
\left|L\left(s, \operatorname{sym}^{2} f\right)\right|^{-1} \leq \exp \left\{D_{-}(\eta)[\log (2 N)]^{\theta(\eta)}\right\}
$$

avec

$$
\theta(\eta):=\frac{\log [(1+\eta) /(1-2 \eta)]}{\log [(1+5 \eta) /(1-2 \eta)]} \quad \text { et } \quad D_{-}(\eta):=\frac{\xi}{\eta^{3}} .
$$

Démonstration. En remplaçant (4.6) par le lemme 10 la preuve est très proche de la preuve du lemme 9. On ne donne donc que les grandes lignes de la preuve. Sur le domaine $D(\eta, N)$ introduit dans la preuve du lemme 9, on définit la fonction

$$
G(s):=\log L\left(s, \operatorname{sym}^{2} f\right)^{-1} .
$$

On déduit de (4.2) que, si $\sigma \geq 4$,

$$
|G(s)|^{2}=\left.\left.|\log | L\left(s, \operatorname{sym}^{2} f\right)\right|^{-1}\right|^{2}+\left|\arg L\left(s, \operatorname{sym}^{2} f\right)^{-1}\right|^{2} \leq C .
$$

D'autre part, pour $\sigma \geq 1+\eta$, on déduit de (4.8) que

$$
\Re e G(s)=\log \left|L\left(s, \operatorname{sym}^{2} f\right)\right|^{-1} \leq \frac{C}{\eta} .
$$

On applique le théorème de Borel-Carathéodory à la fonction $G(s)$ avec

$$
s^{\prime}:=4+i \tau, \quad R^{\prime}:=3-\eta \quad \text { et } \quad r^{\prime}:=3-2 \eta .
$$

En utilisant (4.11) et (4.12), on a

$$
\begin{aligned}
\max _{\left|s-s^{\prime}\right|=r^{\prime}}|G(s)| & \leq \frac{2 r^{\prime}}{R^{\prime}-r^{\prime}} \max _{\left|s-s^{\prime}\right|=R^{\prime}} \Re e G(s)+\frac{R^{\prime}+r^{\prime}}{R^{\prime}-r^{\prime}}\left|G\left(s^{\prime}\right)\right| \\
& \leq \frac{C}{\eta} \cdot \frac{14-4 \eta}{\eta}+\frac{C}{\eta}(6-3 \eta) \\
& \leq \frac{C}{\eta^{2}}
\end{aligned}
$$


En particulier pour $s=\sigma+i \tau$ avec $1+2 \eta \leq \sigma \leq 7-2 \eta$ et $\tau \in \mathbb{R}$, on a

$$
|G(s)| \leq \frac{C}{\eta^{2}}
$$

On applique le théorème de Borel-Carathéodory à $G(s)$ avec

$$
s^{\prime \prime}:=4+i \tau\left(\text { où }|\tau| \leq[\log (2 N)]^{3}\right), \quad R^{\prime \prime}:=3+6 \eta \quad \text { et } \quad r^{\prime \prime}:=3+5 \eta .
$$

En utilisant le lemme 10 et (4.2), on a

$$
\begin{aligned}
\max _{\left|s-s^{\prime \prime}\right|=r^{\prime \prime}}|G(s)| & \leq \frac{2 r^{\prime \prime}}{R^{\prime \prime}-r^{\prime \prime}} \max _{\left|s-s^{\prime \prime}\right|=R^{\prime \prime}} \Re e G(s)+\frac{R^{\prime \prime}+r^{\prime \prime}}{R^{\prime \prime}-r^{\prime \prime}}\left|G\left(s^{\prime \prime}\right)\right| \\
& \leq \xi \frac{6+10 \eta}{\eta^{2}}\left[\frac{1}{\eta}+\log (2 N)\right]+C \frac{6+11 \eta}{\eta} \\
& \leq \frac{\xi}{\eta^{2}}\left[\frac{1}{\eta}+\log (2 N)\right] .
\end{aligned}
$$

En particulier pour $s=\sigma+i \tau$ avec $1-5 \eta \leq \sigma \leq 7+5 \eta$ et $|\tau| \leq[\log (2 N)]^{3}$, on a

$$
|G(s)| \leq \frac{\xi}{\eta^{2}}\left[\frac{1}{\eta}+\log (2 N)\right] .
$$

Maintenant on applique le théorème des trois cercles de Hadamard à $G(s)$ en choisissant trois cercles de même centre $s_{0}=2+i \tau\left(\operatorname{avec}|\tau| \leq[\log (2 N)]^{2}\right)$ et de rayons

$$
r_{1}:=1-2 \eta, \quad r_{2}:=2-\sigma \quad(\text { où } 1-\eta \leq \sigma<1), \quad r_{3}:=1+5 \eta .
$$

En posant

$$
M(r):=\max _{\left|s-s_{0}\right|=r}|G(s)|
$$

on a

$$
M\left(r_{2}\right) \leq M\left(r_{1}\right)^{1-a} M\left(r_{3}\right)^{a}
$$

où $a:=\log \left(r_{2} / r_{1}\right) / \log \left(r_{3} / r_{1}\right)$. En majorant $M\left(r_{1}\right)$ et $M\left(r_{3}\right)$ par (4.13) et (4.14) respectivement, on obtient

$$
M\left(r_{2}\right) \leq\left(\frac{C}{\eta^{2}}\right)^{1-a}\left\{\frac{\xi}{\eta^{2}}\left[\frac{1}{\eta}+\log (2 N)\right]\right\}^{a}
$$

qui implique le résultat souhaité. 


\subsection{Formules de traces pour les puissances de $L\left(s, \operatorname{sym}^{2} f\right)$}

L'objet de cette partie est de donner des formules de type «Petersson» pour les coefficients de Fourier de $L\left(s, \operatorname{sym}^{2} f\right)^{\ell}$ puis d'étudier les termes principaux et d'erreur de ces formules.

\subsubsection{Puissances positives}

On donne une formule de Petersson pour $\rho_{f}^{(+\ell)}$ avec $\ell \in \mathbb{N}^{*}$.

Lemme 12. Soit $k>0$ un entier pair. Soient $N$ un entier sans facteur carré et $n, \ell \in \mathbb{N}^{*}$. Alors on a

$$
\sum_{f \in \mathrm{H}_{k}^{*}(N)} \omega(f) \rho_{f}^{(+\ell)}(n)=\frac{\varphi(N)}{N} m_{+\ell}^{(N)}(n)+O_{k}\left(\frac{\tau(N)^{2}}{N} n^{1 / 2} r_{+\ell}(n) \log (2 n N)\right)
$$

où

et

$$
m_{+\ell}^{(N)}(n):=\sum_{\substack{\mathbf{a}, \mathbf{b}, \mathbf{c} \in \mathbb{N}^{\ell} \\\left|\mathbf{a}^{2} \mathbf{b} \mathbf{c}\right|=n}} \mathbb{1}^{(N)}(|\mathbf{a b}|) \frac{\mathbb{1}_{N}(|\mathbf{c}|)}{|\mathbf{c}|} \sum_{\substack{\mathbf{d} \in \mathcal{E}_{\ell}(\mathbf{b}) \\|\mathbf{d}|=|\mathbf{b}|}} 1
$$

$$
r_{+\ell}(n):=\sum_{\substack{\mathbf{a}, \mathbf{b}, \mathbf{c} \in \mathbb{N}^{\ell} \\\left|\mathbf{a}^{2} \mathbf{b c}\right|=n}} \mathbb{1}^{(N)}(|\mathbf{a b}|) \frac{\mathbb{1}_{N}(|\mathbf{c}|)}{|\mathbf{c}|} \sum_{\substack{\mathbf{d} \in \mathbb{N}^{\ell} \\ d_{j} \mid b_{j}^{2}(1 \leq j \leq \ell)}} 1 .
$$

La constante impliquée dans le symbole $O$ ne dépend que de $k$.

Démonstration. D'après (3.20), on trouve

$$
\sum_{f \in \mathrm{H}_{k}^{*}(N)} \omega(f) \rho_{f}^{(+\ell)}(n)=\sum_{\substack{\mathbf{a}, \mathbf{b}, \mathbf{c} \in \mathbb{N}^{\ell} \\\left|\mathbf{a}^{2} \mathbf{b} \mathbf{c}\right|=n}} \mathbb{1}^{(N)}(|\mathbf{a b}|) \frac{\mathbb{1}_{N}(|\mathbf{c}|)}{|\mathbf{c}|} \sum_{\mathbf{d} \in \mathcal{E}_{\ell}(\mathbf{b})} \sum_{f \in \mathrm{H}_{k}^{*}(N)} \omega(f) \lambda_{f}\left(\frac{|\mathbf{b}|^{2}}{|\mathbf{d}|^{2}}\right) .
$$

En remarquant que seuls contribuent les b tels que $(|\mathbf{b}|, N)=1$, on peut appliquer le lemme 4 à la somme intérieure avec $(m, n)=\left(1,|\mathbf{b}|^{2} /|\mathbf{d}|^{2}\right)$. La contribution du terme principal est $\varphi(N) N^{-1} m_{+\ell}^{(N)}(n)$ et celle du terme d'erreur est

$$
\begin{aligned}
& \ll \frac{\tau(N)^{2}}{N} \sum_{\substack{\mathbf{a}, \mathbf{b}, \mathbf{c} \in \mathbb{N}^{\ell} \\
\left|\mathbf{a}^{2} \mathbf{b} \mathbf{c}\right|=n}} \mathbb{1}^{(N)}(|\mathbf{a b}|) \frac{\mathbb{1}_{N}(|\mathbf{c}|)}{|\mathbf{c}|} \sum_{\mathbf{d} \in \mathcal{E}_{\ell}(\mathbf{b})} \frac{|\mathbf{b}|^{1 / 2}}{|\mathbf{d}|^{1 / 2}} \log \left(2 N \frac{|\mathbf{b}|^{2}}{|\mathbf{d}|^{2}}\right) \\
& \ll \frac{\tau(N)^{2}}{N} n^{1 / 2} \log (2 n N) \sum_{\substack{\mathbf{a}, \mathbf{b}, \mathbf{c} \in \mathbb{N}^{\ell} \\
\left|\mathbf{a}^{2} \mathbf{b} \mathbf{c}\right|=n}} \frac{\mathbb{1}^{(N)}(|\mathbf{a b}|) \mathbb{1}_{N}(|\mathbf{c}|)}{|\mathbf{a}||\mathbf{c}|^{3 / 2}} \sum_{\substack{\mathbf{d} \in \mathbb{N}^{\ell-1} \\
d_{j-1} \mid b_{j}^{2}(2 \leq j \leq \ell)}} \frac{1}{|\mathbf{d}|^{1 / 2}} .
\end{aligned}
$$

Puisque la dernière double somme est majorée par $r_{+\ell}(n)$, cela achève la démonstration. 
Remarque 13. Le remplacement de la dernière double somme par $r_{+\ell}(n)$ a pour but de faciliter les calculs dans la suite, sans perdre d'informations essentielles.

Pour la suite, on a besoin d'introduire les entiers $m_{+\ell, \nu}$. Si $p \in \mathcal{P}$ ne divise pas $N$ et si $\nu \geq 0$, on pose

$$
m_{+\ell, \nu}:=m_{+\ell}^{(N)}\left(p^{\nu}\right) .
$$

Ces nombres ne dépendent ni de $p$, ni de $N$.

On étudie maintenant la série de Dirichlet issue du terme principal de la formule du lemme 12 .

Lemme 14. Soient $N$ un entier sans facteur carré et $\ell \in \mathbb{N}^{*}$. Pour $\sigma>\frac{1}{2}$, on $a$

$$
\sum_{n \geq 1} m_{+\ell}^{(N)}(n) n^{-s}=\zeta^{(N)}(2 s)^{\ell(\ell+1) / 2} \mathcal{M}_{+\ell}^{(N)}(s)
$$

où

$$
\mathcal{M}_{+\ell}^{(N)}(s):=\sum_{n \geq 1} \widetilde{m}_{+\ell}^{(N)}(n) n^{-s}
$$

est une série de Dirichlet absolument convergente pour $\sigma>\frac{1}{3}$. En particulier, pour tout $\varepsilon>0, \sigma \geq \frac{1}{2}+\varepsilon$ et $\ell \geq 2$ on a

$$
\left|\mathcal{M}_{+\ell}^{(N)}(s)\right| \leq \sum_{n \geq 1}\left|\widetilde{m}_{+\ell}^{(N)}(n)\right| n^{-1 / 2-\varepsilon} \leq e^{\iota(\varepsilon) \ell^{2}}
$$

où $\iota(\varepsilon)$ est une constante ne dépendant que de $\varepsilon$.

Démonstration. On prouve l'existence de $\mathcal{M}_{+\ell}^{(N)}$. En posant

$$
\widehat{m}_{+\ell}^{(N)}(n):=\mathbb{1}^{(N)}(n) \sum_{\substack{\mathbf{b} \in \mathbb{N}^{\ell} \\|\mathbf{b}|=n}} \sum_{\substack{\mathbf{d} \in \mathcal{E}_{\ell}(\mathbf{b}) \\|\mathbf{d}|=|\mathbf{b}|}} 1
$$

on a

$$
m_{+\ell}^{(N)}(n)=\underbrace{\mu_{N}^{+} * \cdots * \mu_{N}^{+}}_{\ell \text { fois }} * \underbrace{\square^{(N)} * \cdots * \square^{(N)}}_{\ell \text { fois }} * \widehat{m}_{+\ell}^{(N)}(n) .
$$

La fonction $n \mapsto \widehat{m}_{+\ell}^{(N)}(n)$ est multiplicative et

$$
\widehat{m}_{+\ell}^{(N)}\left(p^{\nu}\right)= \begin{cases}0 & \text { si } p \mid N ; \\ \widehat{m}_{+\ell, \nu} & \text { si } p \nmid N,\end{cases}
$$


où

$$
\widehat{m}_{+\ell, \nu}:=\sum_{\substack{\boldsymbol{\beta} \in \mathbb{N}^{\ell} \\ \operatorname{tr} \boldsymbol{\beta}=\nu}} \sum_{\substack{\boldsymbol{\delta} \in \mathbb{N}^{\ell-1}, \operatorname{tr} \boldsymbol{\delta}=\nu \\ \delta_{j} \leq 2}} 1 .
$$

En posant

$$
\widehat{r}_{+\ell, \nu}:=\sum_{\substack{\boldsymbol{\nu} \in \mathbb{N}^{\ell} \\ \operatorname{tr} \boldsymbol{\nu}=\nu}}\left(2 \nu_{1}+1\right) \cdots\left(2 \nu_{\ell}+1\right)
$$

on a

$$
\widehat{m}_{+\ell, \nu} \leq \widehat{r}_{+\ell, \nu}, \quad \widehat{r}_{+\ell, 1}=3 \ell, \widehat{r}_{+\ell, 2}=\frac{\ell(9 \ell+1)}{2} .
$$

On vérifie que $\widehat{m}_{+\ell, 1}=0, \widehat{m}_{+\ell, 2}=\ell(\ell-1) / 2$. Pour $\sigma>\frac{1}{2}$, on peut donc écrire

$$
\begin{aligned}
\sum_{n \geq 1} m_{+\ell}^{(N)}(n) n^{-s} & =\zeta^{(N)}(2 s)^{\ell} \prod_{p \nmid N} \sum_{\nu \geq 0} \widehat{m}_{+\ell, \nu} p^{-\nu s} \prod_{p \mid N}\left(1-p^{-s-1}\right)^{-\ell} \\
& =\zeta^{(N)}(2 s)^{\ell(\ell+1) / 2} \mathcal{M}_{+\ell}^{(N)}(s),
\end{aligned}
$$

avec

$$
\begin{aligned}
\mathcal{M}_{+\ell}^{(N)}(s) & :=\prod_{p \mid N}\left(1-p^{-s-1}\right)^{-\ell} \prod_{p \nmid N}\left[\left(1-p^{-2 s}\right)^{\widehat{m}_{+\ell, 2}} \sum_{\nu \geq 0} \widehat{m}_{+\ell, \nu} p^{-\nu s}\right] \\
& :=\prod_{p} \sum_{\nu \geq 0} \widetilde{m}_{+\ell}^{(N)}\left(p^{\nu}\right) p^{-\nu s} .
\end{aligned}
$$

On étudie ensuite la convergence de la série $\mathcal{M}_{+\ell}^{(N)}(s)$. Puisque $\widehat{m}_{+\ell, 1}=0$, pour $p \nmid N$ on a

$$
\begin{aligned}
\sum_{\nu \geq 0} \frac{\widetilde{m}_{+\ell}^{(N)}\left(p^{\nu}\right)}{p^{\nu s}}=1 & -\frac{\widehat{m}_{+\ell, 2}^{2}}{p^{4 s}}+\left(1+\frac{\widehat{m}_{+\ell, 2}}{p^{2 s}}\right) \sum_{2 \leq j \leq \widehat{m}_{+\ell, 2}}\left(\begin{array}{c}
\widehat{m}_{+\ell, 2} \\
j
\end{array}\right) \frac{(-1)^{j}}{p^{2 j s}} \\
& +\left(1-\frac{1}{p^{2 s}}\right)^{\widehat{m}_{+\ell, 2}} \sum_{\nu \geq 3} \frac{\widehat{m}_{+\ell, \nu}}{p^{\nu s}}
\end{aligned}
$$

puis, pour $\sigma \geq \frac{1}{2}$,

$$
\begin{aligned}
\sum_{\nu \geq 0} \frac{\left|\widetilde{m}_{+\ell}^{(N)}\left(p^{\nu}\right)\right|}{p^{\nu \sigma}} \leq 1 & +\frac{\widehat{m}_{+\ell, 2}^{2}}{p^{2}}+\left(1+\frac{\widehat{m}_{+\ell, 2}}{p}\right) \sum_{2 \leq j \leq \widehat{m}_{+\ell, 2}}\left(\begin{array}{c}
\widehat{m}_{+\ell, 2} \\
j
\end{array}\right) \frac{1}{p^{j}} \\
& +\left(1+\frac{1}{p}\right)^{\widehat{m}_{+\ell, 2}} \sum_{\nu \geq 3} \frac{\widehat{m}_{+\ell, \nu}}{p^{\nu / 2}} .
\end{aligned}
$$

Ainsi, la série de Dirichlet $\mathcal{M}_{+\ell}^{(N)}(s)$ converge absolument pour $\sigma>\frac{1}{3}$. 
Enfin, on majore

$$
\sum_{n}\left|\widetilde{m}_{+\ell}^{(N)}(n)\right| n^{-\sigma}
$$

pour $\sigma \geq \frac{1}{2}+\varepsilon$. De la première inégalité de (4.18), on déduit

$$
\begin{aligned}
\sum_{\nu \geq 0} \frac{\widehat{m}_{+\ell, \nu}}{p^{\nu / 2}} & \leq \sum_{\nu \geq 0} \sum_{\substack{\boldsymbol{\nu} \in \mathbb{N}^{\ell} \\
\operatorname{tr} \boldsymbol{\nu}=\nu}} \frac{\left(2 \nu_{1}+1\right) \cdots\left(2 \nu_{\ell}+1\right)}{p^{\nu_{1} / 2} \cdots p^{\nu_{\ell} / 2}} \\
& =\left(\sum_{\nu \geq 0} \frac{2 \nu+1}{p^{\nu / 2}}\right)^{\ell}=\left[\frac{1+p^{-1 / 2}}{\left(1-p^{-1 / 2}\right)^{2}}\right]^{\ell} .
\end{aligned}
$$

De (4.19) et (4.21) on déduit

$$
\begin{aligned}
\prod_{p \leq \ell^{2}} \sum_{\nu \geq 0} \frac{\left|\widetilde{m}_{+\ell}^{(N)}\left(p^{\nu}\right)\right|}{p^{\nu(1 / 2+\varepsilon)}} & \leq \prod_{p \leq \ell^{2}}\left(1-\frac{1}{p^{3 / 2}}\right)^{-\ell}\left(1+\frac{1}{p^{1+\varepsilon}}\right)^{\widehat{m}_{+\ell, 2}}\left[\frac{1+p^{-1 / 2}}{\left(1-p^{-1 / 2}\right)^{2}}\right]^{\ell} \\
& \leq e^{\ell(\varepsilon) \ell^{2}} .
\end{aligned}
$$

En utilisant (4.18), on peut aussi écrire

$$
\begin{aligned}
\sum_{\nu \geq 3} \frac{\widehat{m}_{+\ell, \nu}}{p^{\nu / 2}} & \leq \sum_{\nu \geq 0} \sum_{\substack{\boldsymbol{\nu} \in \mathbb{N}^{\ell} \\
\operatorname{tr} \boldsymbol{\nu}=\nu}} \frac{\left(2 \nu_{1}+1\right) \cdots\left(2 \nu_{\ell}+1\right)}{p^{\nu_{1} / 2} \cdots p^{\nu_{\ell} / 2}}-1-\frac{3 \ell}{p^{1 / 2}}-\frac{\ell(9 \ell+1)}{2 p} \\
& =\left[\frac{1+p^{-1 / 2}}{\left(1-p^{-1 / 2}\right)^{2}}\right]^{\ell}-1-\frac{3 \ell}{p^{1 / 2}}-\frac{\ell(9 \ell+1)}{2 p} .
\end{aligned}
$$

Pour $|x \ell| \leq 1$, on a

$$
\begin{aligned}
{\left[\frac{1+x}{(1-x)^{2}}\right]^{\ell} } & =\left[1+3 x+5 x^{2}+O\left(x^{3}\right)\right]^{\ell} \\
& =1+3 \ell x+\frac{\ell(9 \ell+1)}{2} x^{2}+O\left((\ell x)^{3}\right),
\end{aligned}
$$

où les constantes impliquées dans le symbole $O$ sont absolues. Choisissant $x=p^{-1 / 2}$ avec $p>\ell^{2}$ on déduit du report de (4.24) dans (4.23) que

$$
\sum_{\nu \geq 3} \frac{\widehat{m}_{+\ell, \nu}}{p^{\nu / 2}} \leq C \frac{\ell^{3}}{p^{3 / 2}} \quad\left(p>\ell^{2}\right) .
$$

Pour $p>\ell^{2}$, on a aussi

$$
\sum_{2 \leq j \leq \widehat{m}_{+\ell, 2}}\left(\begin{array}{c}
\widehat{m}_{+\ell, 2} \\
j
\end{array}\right) \frac{1}{p^{j}} \leq \sum_{j \geq 2} \frac{\left(\ell^{2} / p\right)^{j}}{j !}=e^{\ell^{2} / p}-1-\frac{\ell^{2}}{p} \leq C \frac{\ell^{4}}{p^{2}} .
$$


En combinant (4.25) et (4.26) avec (4.20), on déduit, pour $p \nmid N$ et $p>\ell^{2}$,

$$
\begin{aligned}
\sum_{\nu \geq 0} \frac{\left|\widetilde{m}_{+\ell}^{(N)}\left(p^{\nu}\right)\right|}{p^{\nu / 2}} & \leq 1+\frac{\widehat{m}_{+\ell, 2}^{2}}{p^{2}}+2 \sum_{2 \leq j \leq \widehat{m}_{+\ell, 2}}\left(\begin{array}{c}
\widehat{m}_{+\ell, 2} \\
j
\end{array}\right) \frac{1}{p^{j}}+e \sum_{\nu \geq 3} \frac{\widehat{m}_{+\ell, \nu}}{p^{\nu / 2}} \\
& \leq 1+C \frac{\ell^{3}}{p^{3 / 2}} .
\end{aligned}
$$

Cette estimation reste vraie pour $p \mid N$ et $p>\ell^{2}$ puisque dans ce cas,

$$
\sum_{\nu \geq 0} \frac{\left|\tilde{m}_{+\ell}^{(N)}\left(p^{\nu}\right)\right|}{p^{\nu / 2}}=\left(1-\frac{1}{p^{3 / 2}}\right)^{-\ell}=1+O\left(\frac{\ell}{p^{3 / 2}}\right) .
$$

Ainsi,

$$
\prod_{p>\ell^{2}} \sum_{\nu \geq 0} \frac{\left|\widetilde{m}_{+\ell}^{(N)}\left(p^{\nu}\right)\right|}{p^{\nu / 2}} \leq \prod_{p>\ell^{2}}\left(1+C \frac{\ell^{3}}{p^{3 / 2}}\right) \leq e^{C \ell^{2}} .
$$

L'inégalité souhaitée découle de (4.22) et (4.27).

On étudie de même la série de Dirichlet issue du terme d'erreur de la formule du lemme 12 .

Lemme 15. Soit $\ell \in \mathbb{N}^{*}$. Pour $\sigma>1$, on a

$$
\sum_{n \geq 1} r_{+\ell}(n) n^{-s}=\zeta^{(N)}(s)^{3 \ell} \mathcal{R}_{+\ell}(s)
$$

où

$$
\mathcal{R}_{+\ell}(s):=\sum_{n \geq 1} \widetilde{r}_{+\ell}(n) n^{-s}
$$

est une série de Dirichlet absolument convergente pour $\sigma>\frac{1}{2}$. En particulier pour $\sigma \geq 1$ et $\ell \geq 2$ on $a$

$$
\left|\mathcal{R}_{+\ell}(s)\right| \leq \sum_{n \geq 1} \frac{\left|\widetilde{r}_{+\ell}(n)\right|}{n} \leq[\log (3 \ell)]^{C \ell}
$$

Démonstration. On prouve l'existence de $\mathcal{R}_{+\ell}$. On pose

$$
\widehat{r}_{+\ell}(n):=\sum_{\substack{\mathbf{b}, \mathbf{c} \in \mathbb{N}^{\ell} \\|\mathbf{b} \mathbf{c}|=n}} \mathbb{1}^{(N)}(|\mathbf{b}|) \frac{\mathbb{1}_{N}(|\mathbf{c}|)}{|\mathbf{c}|} \sum_{\substack{\mathbf{d} \in \mathbb{N}^{\ell} \\ d_{j} \mid b_{j}^{2}(1 \leq j \leq \ell)}} 1 .
$$


Alors on a

$$
r_{+\ell}=\underbrace{\square^{(N)} * \cdots * \square^{(N)}}_{\ell \text { fois }} * \widehat{r}_{+\ell} .
$$

La fonction $\widehat{r}_{+\ell}(n)$ est multiplicative en $n$ et pour $p \nmid N$ on a

$$
\widehat{r}_{+\ell}\left(p^{\nu}\right)=\widehat{r}_{+\ell, \nu}
$$

D'autre part, si $p \mid N$, alors

$$
\widehat{r}_{+\ell}\left(p^{\nu}\right)=\frac{1}{p^{\nu}} \sum_{\substack{\nu_{1}, \ldots, \nu_{\ell} \in \mathbb{N}^{\ell} \\ \nu_{1}+\cdots+\nu_{\ell}=\nu}} 1 .
$$

Pour tout $s \in \mathbb{C}$ tel que $\sigma>1$, on peut donc écrire

$$
\begin{aligned}
\sum_{n \geq 1} \frac{r_{+\ell}(n)}{n^{s}} & =\zeta^{(N)}(2 s)^{\ell} \prod_{p \mid N}\left(1-\frac{1}{p^{s+1}}\right)^{-\ell} \prod_{p \nmid N} \sum_{\nu \geq 0} \frac{\widehat{r}_{+\ell, \nu}}{p^{\nu s}} \\
& =\zeta^{(N)}(s)^{3 \ell} \mathcal{R}_{+\ell}(s),
\end{aligned}
$$

où

$$
\begin{aligned}
\mathcal{R}_{+\ell}(s) & :=\zeta^{(N)}(2 s)^{\ell} \prod_{p \mid N}\left(1-\frac{1}{p^{s+1}}\right)^{-\ell} \prod_{p \nmid N}\left(1-\frac{1}{p^{s}}\right)^{3 \ell} \sum_{\nu \geq 0} \frac{\widehat{r}_{+\ell, \nu}}{p^{\nu s}} \\
& =: \prod_{p} \sum_{\nu \geq 0} \frac{\widetilde{r}_{+\ell}\left(p^{\nu}\right)}{p^{\nu s}} .
\end{aligned}
$$

Puisque $\widetilde{r}_{+\ell}(p)=0$ pour tout $p \nmid N$, la série $\mathcal{R}_{+\ell}(s)$ converge absolument pour $\sigma>\frac{1}{2}$.

Il reste à majorer

$$
\sum_{n \geq 1}\left|\widetilde{r}_{+\ell}(n)\right| n^{-\sigma}
$$

pour $\sigma \geq 1$. D'abord, comme en (4.21) on a

$$
\sum_{\nu \geq 0} \frac{\widehat{r}_{+\ell, \nu}}{p^{\nu}}=\left[\frac{1+p^{-1}}{\left(1-p^{-1}\right)^{2}}\right]^{\ell} .
$$

Pour $\sigma \geq 1$, la contribution des $p \leq \ell$ à (4.28) est donc

$$
\begin{aligned}
\prod_{p \leq \ell} \sum_{\nu \geq 0} \frac{\left|\widetilde{r}_{+\ell}\left(p^{\nu}\right)\right|}{p^{\nu \sigma}} & \leq \prod_{p \leq \ell}\left(1-\frac{1}{p^{2}}\right)^{-2 \ell}\left(1+\frac{1}{p}\right)^{3 \ell}\left[\frac{1+p^{-1}}{\left(1-p^{-1}\right)^{2}}\right]^{\ell} \\
& \leq e^{C \ell \log _{2}(3 \ell)} .
\end{aligned}
$$


On traîte maintenant le cas des premiers $p>\ell$. Puisque $\widehat{r}_{+\ell, 1}=3 \ell$, on a

$$
\begin{aligned}
& \left(1-\frac{1}{p^{s}}\right)^{3 \ell} \sum_{\nu \geq 0} \frac{\widehat{r}_{+\ell, \nu}}{p^{\nu s}}= \\
& =1-\frac{9 \ell^{2}}{p^{2 s}}+\left(1+\frac{3 \ell}{p^{s}}\right) \sum_{2 \leq j \leq 3 \ell}\left(\begin{array}{c}
3 \ell \\
j
\end{array}\right) \frac{(-1)^{j}}{p^{j s}}+\left(1-\frac{1}{p^{s}}\right)^{3 \ell} \sum_{\nu \geq 2} \frac{\widehat{r}_{+\ell, \nu}}{p^{\nu s}} .
\end{aligned}
$$

De plus, on a

$$
\sum_{\nu \geq 2} \frac{\widehat{r}_{+\ell, \nu}}{p^{\nu}}=\left[\frac{1+p^{-1}}{\left(1-p^{-1}\right)^{2}}\right]^{\ell}-1-\frac{3 \ell}{p} \ll \frac{\ell^{2}}{p^{2}}
$$

et, toujours pour $\ell<p$

$$
\sum_{j=2}^{3 \ell}\left(\begin{array}{c}
3 \ell \\
j
\end{array}\right) \frac{1}{p^{j}} \leq \sum_{j \geq 2} \frac{(3 \ell / p)^{j}}{j !} \ll \frac{\ell^{2}}{p^{2}} .
$$

En combinant (4.31) et (4.32) avec (4.30), on a pour $\sigma \geq 1$ l'inégalité

$$
\begin{aligned}
\left(1+\frac{1}{p^{\sigma}}\right)^{3 \ell} \sum_{\nu \geq 0} \frac{\left|\widehat{r}_{+\ell, \nu}\right|}{p^{\nu \sigma}} & \leq 1+\frac{9 \ell^{2}}{p^{2}}+\left(1+\frac{3 \ell}{p}\right) \sum_{j=2}^{3 \ell}\left(\begin{array}{c}
3 \ell \\
j
\end{array}\right) \frac{1}{p^{j}}+\left(1+\frac{1}{p}\right)^{3 \ell} \sum_{\nu \geq 2} \frac{\widehat{r}_{+\ell, \nu}}{p^{\nu / 2}} \\
& \leq 1+C \frac{\ell^{2}}{p^{2}} .
\end{aligned}
$$

Pour $\sigma>1$, la contribution à (4.28) des premiers $p>\ell$ est donc

$$
\prod_{p>\ell} \sum_{\nu \geq 0} \frac{\left|\widetilde{r}_{+\ell}\left(p^{\nu}\right)\right|}{p^{\nu \sigma}} \leq \prod_{p>\ell}\left(1-\frac{1}{p^{2}}\right)^{-2 \ell}\left(1+C \frac{\ell^{2}}{p^{2}}\right) \leq e^{C \ell} .
$$

L'inégalité recherchée résulte de (4.29) et (4.33).

On peut alors majorer l'ordre moyen de la fonction $r_{+\ell}$ dans le

Lemme 16. Il existe un réel $C>0$ tel que, pour tous $t \geq 1$ et $\ell \in \mathbb{N}^{*}$, on a

$$
\sum_{n \leq t} r_{+\ell}(n) \leq t[\log (e t)]^{3 \ell-1}[\log (3 \ell)]^{C \ell}
$$

Démonstration. D'après le lemme 15 , on a $r_{+\ell}=\tau_{3 \ell}^{(N)} * \widetilde{r}_{+\ell}$ où $\tau_{\ell}^{(N)}$ est définie par

$$
\prod_{p \nmid N}\left(1-\frac{1}{p^{s}}\right)^{-\ell}=\sum_{n \geq 1} \frac{\tau_{\ell}^{(N)}(n)}{n^{s}} \quad(\sigma>1) .
$$


Puisque $\tau_{3 \ell}^{(N)}(n) \leq \tau_{3 \ell}(n)$ pour tout $n \geq 1$, il en résulte que

$$
\sum_{n \leq t} r_{+\ell}(n) \leq \sum_{d \leq t}\left|\widetilde{r}_{+\ell}(d)\right| \sum_{m \leq t / d} \tau_{3 \ell}(m) \leq t[\log (e t)]^{3 \ell-1}[\log (3 \ell)]^{C \ell}
$$

Cela achève la démonstration.

\subsubsection{Puissances négatives}

Ce paragraphe est très semblable au paragraphe 4.2.1. On y donne une formule de Petersson pour $\rho_{f}^{(-\ell)}(n)$ avec $\ell \in \mathbb{N}^{*}$.

Lemme 17. Soient $N$ un entier sans facteur carré et $n, \ell \in \mathbb{N}^{*}$. Alors on a

$$
\sum_{f \in \mathrm{H}_{k}^{*}(N)} \omega(f) \rho_{f}^{(-\ell)}(n)=\frac{\varphi(N)}{N} m_{-\ell}^{(N)}(n)+O_{k}\left(\frac{\tau(N)^{2}}{N} n^{1 / 2} r_{-\ell}(n) \log (2 n N)\right),
$$

où

$$
m_{-\ell}^{(N)}(n):=\sum_{\substack{\mathbf{a}, \mathbf{b}, \mathbf{c}, \mathbf{e} \in \mathbb{N}^{\ell} \\\left|\mathbf{a} \mathbf{b}^{2} \mathbf{c}^{3} \mathbf{e}\right|=n}} \mathbb{1}^{(N)}(|\mathbf{a b c}|) \frac{\mathbb{1}_{N}(|\mathbf{e}|)}{|\mathbf{e}|} \prod_{j=1}^{\ell} \mu\left(a_{j} b_{j} c_{j}\right) \mu\left(b_{j}\right) \mu\left(e_{j}\right) \sum_{\substack{\mathbf{d} \in \mathcal{E}_{\ell}(\mathbf{a b}) \\|\mathbf{d}|=|\mathbf{a b}|}} 1
$$

et

$$
r_{-\ell}(n):=\sum_{\substack{\mathbf{a}, \mathbf{b}, \mathbf{c}, \mathbf{e} \in \mathbb{N}^{\ell} \\\left|\mathbf{a} \mathbf{b}^{2} \mathbf{c}^{3} \mathbf{e}\right|=n}} \mathbb{1}^{(N)}(|\mathbf{a b c}|) \frac{\mathbb{1}_{N}(|\mathbf{e}|)}{|\mathbf{e}|}\left|\prod_{j=1}^{\ell} \mu\left(a_{j} b_{j} c_{j}\right) \mu\left(e_{j}\right)\right| \sum_{\substack{\mathbf{d} \in \mathbb{N}^{\ell} \\ d_{j} \mid\left(a_{j} b_{j}\right)^{2}(1 \leq j \leq \ell)}} 1 .
$$

La constante impliquée dans le symbole $O$ ne dépend que de $k$.

Démonstration. D'après (3.21), la somme à évaluer est égale à

$$
\begin{aligned}
\sum_{\substack{\mathbf{a}, \mathbf{b}, \mathbf{c}, \mathbf{e} \in \mathbb{N}^{\ell} \\
\left|\mathbf{a} \mathbf{b}^{2} \mathbf{c}^{3} \mathbf{e}\right|=n}} \mathbb{1}^{(N)}(|\mathbf{a b c}|) \frac{\mathbb{1}_{N}(|\mathbf{e}|)}{|\mathbf{e}|}\left[\prod_{j=1}^{\ell} \mu\left(a_{j} b_{j} c_{j}\right) \mu\left(b_{j}\right) \mu\left(e_{j}\right)\right] \\
\times \sum_{\mathbf{d} \in \mathcal{E}_{\ell}(\mathbf{a b})} \sum_{f \in \mathrm{H}_{k}^{*}(N)} \omega(f) \lambda_{f}\left(\frac{|\mathbf{a b}|^{2}}{|\mathbf{d}|^{2}}\right) .
\end{aligned}
$$

En remarquant que seuls contribuent les $\mathbf{a}$, $\mathbf{b}$ tels que $(|\mathbf{a b}|, N)=1$ on peut appliquer le lemme 4 à la somme intérieure avec $(m, n)=\left(1,|\mathbf{a b}|^{2} /|\mathbf{d}|^{2}\right)$. 
La contribution du terme principal est $\varphi(N) N^{-1} m_{-\ell}^{(N)}(n)$ et celle du terme d'erreur est

$$
\begin{aligned}
& \ll \frac{\tau(N)^{2}}{N} \sum_{\substack{\mathbf{a}, \mathbf{b}, \mathbf{c}, \mathbf{e} \in \mathbb{N}^{\ell} \\
\left|\mathbf{a b}^{2} \mathbf{c}^{3} \mathbf{e}\right|=n}} \mathbb{1}_{N}(|\mathbf{a b c}|) \frac{\mathbb{1}_{N}(|\mathbf{e}|)}{|\mathbf{e}|}\left|\prod_{j=1}^{\ell} \mu\left(a_{j} b_{j} c_{j}\right) \mu\left(e_{j}\right)\right| \\
& \times \sum_{\substack{\mathbf{d} \in \mathcal{E}_{\ell}(\mathbf{a b}) \\
|\mathbf{d}|^{1 / 2}}} \frac{|\mathbf{a b}|^{1 / 2}}{\mid} \log \left(2 N \frac{|\mathbf{a b}|^{2}}{|\mathbf{d}|^{2}}\right) \\
& \ll \frac{\tau(N)^{2}}{N} n^{1 / 2} \log (2 n N) \sum_{\substack{\mathbf{a}, \mathbf{b}, \mathbf{c}, \mathbf{e} \in \mathbb{N}^{\ell} \\
\left|\mathbf{a b} \mathbf{b}^{2} \mathbf{c}^{3} \mathbf{e}\right|=n}} \mathbb{1}^{(N)}(|\mathbf{a b c}|) \frac{\mathbb{1}_{N}(|\mathbf{e}|)}{|\mathbf{e}|}\left|\mathbf{b c}^{3} \mathbf{e}\right|^{-1 / 2} \\
& \times\left|\prod_{j=1}^{\ell} \mu\left(a_{j} b_{j} c_{j}\right) \mu\left(e_{j}\right)\right| \sum_{\substack{\mathbf{d} \in \mathbb{N}^{\ell-1} \\
d_{j-1} \mid\left(a_{j} b_{j}\right)^{2}(2 \leq j \leq \ell)}} 1 .
\end{aligned}
$$

Puisque la dernière double somme est majorée par $r_{-\ell}(n)$, cela achève la démonstration.

Remarque 18. Le remplacement de la dernière double somme par $r_{-\ell}(n)$ a pour but de faciliter les calculs de la suite, sans perdre d'informations essentielles.

Pour la suite, on a besoin d'introduire les entiers $m_{-\ell, \nu}$. Si $p \in \mathcal{P}$ ne divise pas $N$ et si $\nu \geq 0$, on pose

$$
m_{-\ell, \nu}:=m_{-\ell}^{(N)}\left(p^{\nu}\right)
$$

Ces nombres ne dépendent ni de $p$, ni de $N$. Il est important de constater que ces nombres sont les mêmes que les nombres $\widehat{m}_{-\ell, \nu}$ définis ci-dessous en (4.37) mais que celà est faux en remplaçant $-\ell$ par $\ell$. La formule (4.17) donne

$$
m_{+\ell, \nu}=\sum_{\substack{\left(\alpha_{1}, \ldots, \alpha_{\ell}, \beta\right) \in \mathbb{N}^{\ell+1} \\ 2\left(\alpha_{1}+\cdots+\alpha_{\ell}\right)+\beta=\nu}} \widehat{m}_{+\ell, \beta}
$$

alors que la formule (4.37) donne

$$
m_{-\ell, \nu}=\widehat{m}_{-\ell, \nu}
$$

On étudie maintenant la série de Dirichlet issue du terme principal de la formule du lemme 17 . 
Lemme 19. Soient $N$ un entier sans facteur carré et $\ell \in \mathbb{N}^{*}$. Pour $\sigma>\frac{1}{2}$, on a

$$
\sum_{n \geq 1} m_{-\ell}^{(N)}(n) n^{-s}=\zeta^{(N)}(2 s)^{\ell(\ell-1) / 2} \mathcal{M}_{-\ell}^{(N)}(s)
$$

où

$$
\mathcal{M}_{-\ell}^{(N)}(s):=\sum_{n \geq 1} \widetilde{m}_{-\ell}^{(N)}(n) n^{-s}
$$

est une série de Dirichlet absolument convergente pour $\sigma>\frac{1}{3}$. En particulier pour tous $\varepsilon>0, \sigma \geq \frac{1}{2}+\varepsilon$ et $\ell \geq 2$ on a

$$
\left|\mathcal{M}_{-\ell}^{(N)}(s)\right| \leq \sum_{n \geq 1}\left|\widetilde{m}_{-\ell}^{(N)}(n)\right| n^{-1 / 2-\varepsilon} \leq e^{\iota(\varepsilon) \ell^{2}}
$$

où $\iota(\varepsilon)>0$ est une constante ne dépendant que de $\varepsilon$.

Démonstration. On prouve l'existence de $\mathcal{M}_{-\ell}^{(N)}$. En posant

$$
\widehat{m}_{-\ell}^{(N)}(n):=\mathbb{1}^{(N)}(n) \sum_{\substack{\mathbf{a}, \mathbf{b}, \mathbf{c} \in \mathbb{N}^{\ell} \\\left|\mathbf{a b}^{2} \mathbf{c}^{3}\right|=n}}\left[\prod_{j=1}^{\ell} \mu\left(a_{j} b_{j} c_{j}\right) \mu\left(b_{j}\right)\right] \sum_{\substack{\mathbf{d} \in \mathcal{E}_{\ell}(\mathbf{a b}) \\|\mathbf{d}|=|\mathbf{a b}|}} 1
$$

on a

$$
m_{-\ell}^{(N)}(n):=\underbrace{\mu_{N}^{-} * \cdots * \mu_{N}^{-}}_{\ell \text { fois }} * \widehat{m}_{-\ell}^{(N)}(n) .
$$

Les fonctions $n \mapsto m_{-\ell}^{(N)}(n)$ et $n \mapsto \widehat{m}_{-\ell}^{(N)}(n)$ sont multiplicatives et pour $p \nmid N$ on a [19]

$$
m_{-\ell}^{(N)}\left(p^{\nu}\right)=\widehat{m}_{-\ell}^{(N)}\left(p^{\nu}\right)=(-1)^{\nu} a_{\ell}(\nu)=: \widehat{m}_{-\ell, \nu},
$$

où

$$
a_{\ell}(\nu):=\sum_{\substack{\boldsymbol{\alpha}, \boldsymbol{\beta}, \boldsymbol{\gamma} \in\{0,1\}^{\ell} \\ 0 \leq \alpha_{i}+\beta_{i}+\gamma_{i} \leq 1 \\ \operatorname{tr}(\boldsymbol{\alpha}+2 \boldsymbol{\beta}+3 \gamma)=\nu}} \sum_{\substack{\boldsymbol{\delta} \in\{0,1,2\}^{\ell-1} \\ \delta_{i} \leq 2 \min \left\{\alpha_{1}+\beta_{1}+\cdots+\alpha_{i}+\beta_{i}-\delta_{1}-\cdots-\delta_{i-1}, \alpha_{i+1}+\beta_{i+1}\right\} \\ \operatorname{tr}(\boldsymbol{\delta})=\operatorname{tr}(\boldsymbol{\alpha}+\boldsymbol{\beta})}} 1 .
$$

En posant

$$
b_{\ell}(\nu):=\sum_{\substack{\boldsymbol{\alpha}, \boldsymbol{\beta}, \boldsymbol{\gamma} \in\{0,1\}^{\ell} \\ 0 \leq \alpha_{i}+\beta_{i}+\gamma_{i} \leq 1 \\ \operatorname{tr}(\boldsymbol{\alpha}+2 \boldsymbol{\beta}+3 \gamma)=\nu}}\left[2\left(\alpha_{1}+2 \beta_{1}+3 \gamma_{1}\right)+1\right] \cdots\left[2\left(\alpha_{\ell}+2 \beta_{\ell}+3 \gamma_{\ell}\right)+1\right]
$$

on a

$$
\begin{gathered}
a_{\ell}(\nu) \leq b_{\ell}(\nu) \\
b_{\ell}(1)=3 \ell, b_{\ell}(2)=\frac{\ell(9 \ell+1)}{2}, \nu>3 \ell \Rightarrow b_{\ell}(\nu)=0 .
\end{gathered}
$$


On vérifie $a_{\ell}(1)=0$ et $a_{\ell}(2)=\ell(\ell-1) / 2$. Pour $\sigma>\frac{1}{2}$, on peut donc écrire

$$
\begin{aligned}
\sum_{n \geq 1} m_{-\ell}^{(N)}(n) n^{-s} & =\prod_{p \mid N}\left(1-p^{-s-1}\right)^{\ell} \prod_{p \nmid N} \sum_{\nu \geq 0} \widehat{m}_{-\ell, \nu} p^{-\nu s} \\
& =\zeta^{(N)}(2 s)^{\ell(\ell-1) / 2} \mathcal{M}_{-\ell}^{(N)}(s),
\end{aligned}
$$

où

$$
\begin{aligned}
\mathcal{M}_{-\ell}^{(N)}(s) & :=\prod_{p \mid N}\left(1-p^{-s-1}\right)^{\ell} \prod_{p \nmid N}\left[\left(1-p^{-2 s}\right)^{\ell(\ell-1) / 2} \sum_{\nu \geq 0} \widehat{m}_{-\ell, \nu} p^{-\nu s}\right] \\
& =: \prod_{p} \sum_{\nu \geq 0} \widetilde{m}_{-\ell}^{(N)}\left(p^{\nu}\right) p^{-\nu s} .
\end{aligned}
$$

On étudie la convergence de $\mathcal{M}_{-\ell}^{(N)}$. Puisque $a_{\ell}(1)=0$, pour $p \nmid N$ on a

$$
\begin{aligned}
\sum_{\nu \geq 0} \frac{\widetilde{m}_{-\ell}^{(N)}\left(p^{\nu}\right)}{p^{\nu s}}= & {\left[1-\frac{a_{\ell}(2)}{p^{2 s}}+\sum_{2 \leq j \leq a_{\ell}(2)}\left(\begin{array}{c}
a_{\ell}(2) \\
j
\end{array}\right) \frac{(-1)^{j}}{p^{2 j s}}\right] } \\
& \times\left[1+\frac{a_{\ell}(2)}{p^{2 s}}+\sum_{\nu \geq 3} \frac{(-1)^{\nu} a_{\ell}(\nu)}{p^{\nu s}}\right] \\
=1 & -\frac{a_{\ell}(2)^{2}}{p^{4 s}}+\left[1+\frac{a_{\ell}(2)}{p^{2 s}}\right] \sum_{2 \leq j \leq a_{\ell}(2)}\left(\begin{array}{c}
a_{\ell}(2) \\
j
\end{array}\right) \frac{(-1)^{j}}{p^{2 j s}} \\
& +\left(1-\frac{1}{p^{2 s}}\right)^{a_{\ell}(2)} \sum_{\nu \geq 3} \frac{(-1)^{\nu} a_{\ell}(\nu)}{p^{\nu s}} .
\end{aligned}
$$

On déduit de (4.41) et de (4.38) que la série de Dirichlet $\mathcal{M}_{-\ell}^{(N)}(s)$ converge absolument pour $\sigma>\frac{1}{3}$.

Il reste à majorer

$$
\sum_{n \geq 1}\left|\widetilde{m}_{-\ell}^{(N)}(n)\right| n^{-\sigma}
$$

pour $\sigma \geq \frac{1}{2}+\varepsilon$. De (4.38), on déduit

$$
\sum_{\nu \geq 0} \frac{a_{\ell}(\nu)}{p^{\nu / 2}} \leq\left[\sum_{\substack{\alpha, \beta, \gamma \in\{0,1\} \\ 0 \leq \alpha+\beta+\gamma \leq 1 \\ \alpha+2 \beta+3 \gamma \leq 3}} \frac{2(\alpha+2 \beta+3 \gamma)+1}{p^{(\alpha+2 \beta+3 \gamma) / 2}}\right]^{\ell} \leq\left(\sum_{0 \leq \nu \leq 3} \frac{2 \nu+1}{p^{\nu / 2}}\right)^{\ell}
$$

$\operatorname{car} \#\{(\alpha, \beta, \gamma) \in\{0,1\}: 0 \leq \alpha+\beta+\gamma \leq 1$ et $\nu=\alpha+2 \beta+3 \gamma\}=1$ si $0 \leq$ $\nu \leq 3$. 
On en déduit

$$
\sum_{\nu \geq 0} \frac{a_{\ell}(\nu)}{p^{\nu / 2}} \leq\left(1+\frac{15}{p^{1 / 2}}\right)^{\ell}
$$

Le report de (4.42) dans (4.40) donne

$$
\prod_{p \leq \ell^{2}} \sum_{\nu \geq 0} \frac{\left|\widetilde{m}_{-\ell}^{(N)}\left(p^{\nu}\right)\right|}{p^{\nu(1 / 2+\varepsilon)}} \leq \prod_{p \mid N}\left(1+\frac{1}{p^{3 / 2}}\right)^{\ell} \prod_{p \leq \ell^{2}}\left(1+\frac{1}{p^{1+\varepsilon}}\right)^{\ell^{2}}\left(1+\frac{15}{p^{1 / 2}}\right)^{\ell}
$$

$$
\leq e^{\iota(\varepsilon) \ell^{2}} \text {. }
$$

En utilisant (4.38), (4.39) et (4.42), on peut aussi écrire

$$
\begin{aligned}
\sum_{\nu \geq 3} \frac{a_{\ell}(\nu)}{p^{\nu / 2}} & \leq \sum_{\nu=0}^{+\infty} \frac{b_{\ell}(\nu)}{p^{\nu / 2}}-1-\frac{3 \ell}{p^{1 / 2}}-\frac{\ell(9 \ell+1)}{2 p} \\
& \leq\left(\sum_{0 \leq \nu \leq 3} \frac{2 \nu+1}{p^{\nu / 2}}\right)^{\ell}-1-\frac{3 \ell}{p^{1 / 2}}-\frac{\ell(9 \ell+1)}{2 p} .
\end{aligned}
$$

En combinant (4.24) avec $x=p^{-1 / 2}$ où $p>\ell^{2}$ et (4.44), on trouve

$$
\sum_{\nu \geq 3} \frac{a_{\ell}(\nu)}{p^{\nu / 2}} \leq C \frac{\ell^{3}}{p^{3 / 2}}
$$

Toujours pour $p>\ell^{2}$, on a

$$
\sum_{2 \leq j \leq a_{\ell}(2)}\left(\begin{array}{c}
a_{\ell}(2) \\
j
\end{array}\right) \frac{1}{p^{j}} \leq \sum_{j \geq 2} \frac{\left(\ell^{2} / p\right)^{j}}{j !}=e^{\ell^{2} / p}-1-\frac{\ell^{2}}{p} \leq C \frac{\ell^{4}}{p^{2}} .
$$

En combinant (4.45) et (4.46) avec (4.41) on a, pour $p \nmid N$ et $p>\ell^{2}$,

$$
\begin{aligned}
\sum_{\nu \geq 0} \frac{\left|\widetilde{m}_{-\ell}^{(N)}\left(p^{\nu}\right)\right|}{p^{\nu / 2}} & \leq 1+\frac{a_{\ell}(2)^{2}}{p^{2}}+2 \sum_{2 \leq j \leq a_{\ell}(2)}\left(\begin{array}{c}
a_{\ell}(2) \\
j
\end{array}\right) \frac{1}{p^{j}}+e \sum_{\nu \geq 3} \frac{a_{\ell}(\nu)}{p^{\nu / 2}} \\
& \leq 1+C \frac{\ell^{3}}{p^{3 / 2}} .
\end{aligned}
$$

Cette estimation reste valable pour $p \mid N$ et $p>\ell^{2}$. Ainsi

$$
\prod_{p>\ell^{2}} \sum_{\nu \geq 0} \frac{\left|\widetilde{m}_{-\ell}^{(N)}\left(p^{\nu}\right)\right|}{p^{\nu / 2}} \leq \prod_{p>\ell^{2}}\left(1+C \frac{\ell^{3}}{p^{3 / 2}}\right) \leq e^{C \ell^{2}} .
$$

L'inégalité souhaitée découle de (4.43) et (4.47). 
On étudie de même la série de Dirichlet issue du terme d'erreur de la formule du lemme 17.

Lemme 20. Soit $\ell \in \mathbb{N}^{*}$. Pour $\sigma>1$, on a

$$
\sum_{n \geq 1} r_{-\ell}(n) n^{-s}=\zeta^{(N)}(s)^{3 \ell} \mathcal{R}_{-\ell}(s)
$$

où $\mathcal{R}_{-\ell}(s):=\sum_{n>1} \widetilde{r}_{-\ell}(n) n^{-s}$ est une série de Dirichlet absolument convergente pour $\sigma>\frac{1}{2}$. En particulier pour $\sigma \geq 1$ et $\ell \geq 2$ on a

$$
\left|\mathcal{R}_{-\ell}(s)\right| \leq \sum_{n \geq 1} \frac{\left|\widetilde{r}_{-\ell}(n)\right|}{n} \leq[\log (3 \ell)]^{C \ell} .
$$

Démonstration. On prouve l'existence de $\mathcal{R}_{-\ell}$. On pose

$$
r_{-\ell, \nu}:=\sum_{\substack{\boldsymbol{\alpha}, \boldsymbol{\beta}, \boldsymbol{\gamma} \in\{0,1\}^{\ell} \\ 0 \leq \alpha_{i}+\beta_{i}+\gamma_{i} \leq 1 \\ \operatorname{tr}(\boldsymbol{\alpha}+2 \boldsymbol{\beta}+3 \gamma)=\nu}}\left(2 \alpha_{1}+2 \beta_{1}+1\right) \cdots\left(2 \alpha_{\ell}+2 \beta_{\ell}+1\right) .
$$

La fonction $n \mapsto r_{-\ell}(n)$ est multiplicative et pour $p \nmid N$ on a

$$
r_{-\ell}\left(p^{\nu}\right)=r_{-\ell, \nu}
$$

D'autre part, si $p \mid N$, alors

$$
r_{-\ell}\left(p^{\nu}\right)=\frac{1}{p^{\nu}} \sum_{\substack{\nu_{1}, \ldots, \nu_{\ell} \in\{0,1\} \\ \nu_{1}+\cdots+\nu_{\ell}=\nu}} 1
$$

de sorte que par interversion des sommes

$$
\sum_{\nu=0}^{+\infty} \frac{r_{-\ell}\left(p^{\nu}\right)}{p^{\nu s}}=\left(1+\frac{1}{p^{s+1}}\right)^{\ell} .
$$

Pour $\sigma>1$, on déduit de (4.48) et (4.49) que

$$
\sum_{n \geq 1} \frac{r_{-\ell}(n)}{n^{s}}=\prod_{p \mid N}\left(1+\frac{1}{p^{s+1}}\right)^{\ell} \prod_{p \nmid N} \sum_{\nu \geq 0} \frac{r_{-\ell, \nu}}{p^{\nu s}}=\zeta^{(N)}(s)^{3 \ell} \mathcal{R}_{-\ell}(s),
$$

où

$$
\begin{aligned}
\mathcal{R}_{-\ell}(s) & :=\prod_{p \mid N}\left(1+\frac{1}{p^{s+1}}\right)^{\ell} \prod_{p \nmid N}\left[\left(1-\frac{1}{p^{s}}\right)^{3 \ell} \sum_{\nu \geq 0} \frac{r_{-\ell, \nu}}{p^{\nu s}}\right] \\
& =: \prod_{p} \sum_{\nu \geq 0} \frac{\widetilde{r}_{-\ell}\left(p^{\nu}\right)}{p^{\nu s}} .
\end{aligned}
$$


Puisque $r_{-\ell, 1}=3 \ell$ et $r_{-\ell, \nu} \leq b_{\ell}(\nu)$, on peut écrire

$$
\begin{aligned}
\left(1-\frac{1}{p^{s}}\right)^{3 \ell} \sum_{\nu \geq 0} \frac{r_{-\ell, \nu}}{p^{\nu s}}=1 & -\frac{9 \ell^{2}}{p^{2 s}}+\left(1+\frac{3 \ell}{p^{s}}\right) \sum_{2 \leq j \leq 3 \ell}\left(\begin{array}{c}
3 \ell \\
j
\end{array}\right) \frac{(-1)^{j}}{p^{j s}} \\
& +\left(1-\frac{1}{p^{s}}\right)^{3 \ell} \sum_{\nu \geq 2} \frac{r_{-\ell, \nu}}{p^{\nu s}}
\end{aligned}
$$

On déduit de (4.51) et (4.39) que la série de Dirichlet $\mathcal{R}_{-\ell}$ converge absolument pour $\sigma>\frac{1}{2}$.

Il reste à majorer

$$
\sum_{n \geq 1}\left|\widetilde{r}_{-\ell}(n)\right| n^{-\sigma}
$$

pour $\sigma \geq 1$. Puisque $r_{-\ell, \nu} \leq b_{\ell}(\nu)$, de même qu'on a obtenu (4.42) on a

$$
\sum_{\nu \geq 0} \frac{r_{-\ell, \nu}}{p^{\nu}} \leq\left(\sum_{0 \leq \nu \leq 3} \frac{2 \nu+1}{p^{\nu}}\right)^{\ell} \leq\left(1+\frac{15}{p}\right)^{\ell}
$$

Donc pour $\sigma \geq 1$, grâce à (4.50) et (4.52) on a

$$
\begin{aligned}
\prod_{p \leq \ell} \sum_{\nu \geq 0} \frac{\left|\widetilde{r}_{-\ell}\left(p^{\nu}\right)\right|}{p^{\nu \sigma}} & \leq \prod_{p \mid N}\left(1+\frac{1}{p^{2}}\right)^{\ell} \prod_{p \leq \ell}\left(1+\frac{15}{p}\right)^{\ell}\left(1+\frac{1}{p}\right)^{3 \ell} \\
& \leq e^{C \ell \log _{2}(3 \ell)} .
\end{aligned}
$$

Pour $p>\ell$, comme en (4.44) on a

$$
\sum_{\nu \geq 2} \frac{r_{-\ell, \nu}}{p^{\nu}} \leq\left(\sum_{0 \leq \nu \leq 3} \frac{2 \nu+1}{p^{\nu}}\right)^{\ell}-1-\frac{3 \ell}{p} \leq C \frac{\ell^{2}}{p^{2}} .
$$

En combinant (4.54) et (4.32) avec (4.51), on déduit, pour $\sigma \geq 1$ et $p>\ell$,

$$
\sum_{\nu \geq 0} \frac{\left|\widetilde{r}_{-\ell}\left(p^{\nu}\right)\right|}{p^{\nu \sigma}} \leq 1+\frac{9 \ell^{2}}{p^{2}}+4 \sum_{2 \leq j \leq 3 \ell}\left(\begin{array}{c}
3 \ell \\
j
\end{array}\right) \frac{1}{p^{j}}+e^{3} \sum_{\nu \geq 2} \frac{r_{-\ell, \nu}}{p^{\nu / 2}} \leq 1+C \frac{\ell^{2}}{p^{2}} .
$$

D'où pour $\sigma \geq 1$

$$
\prod_{p>\ell} \sum_{\nu \geq 0} \frac{\left|\widetilde{r}_{-\ell}\left(p^{\nu}\right)\right|}{p^{\nu \sigma}} \leq \prod_{p>\ell}\left(1+C \frac{\ell^{2}}{p^{2}}\right) \leq e^{C \ell} .
$$

L'inégalité souhaitée découle de (4.53) et (4.55). 
De même qu'on a montré le lemme 16, on peut montrer le

Lemme 21. Il existe un réel $C>0$ tel que, pour tout $t \geq 1$ et $\ell \in \mathbb{N}^{*}$, on a

$$
\sum_{n \leq t} r_{-\ell}(n) \leq t[\log (e t)]^{3 \ell-1}[\log (3 \ell)]^{C \ell}
$$

\subsection{Preuve du théorème $B$}

Si $f \in \mathrm{H}_{k}^{*}(N)$, on définit pour tous $\ell \in \mathbb{N}^{*}$ et $x \geq 1$ la somme courte

$$
\omega_{f}^{( \pm \ell)}(x):=\sum_{n=1}^{+\infty} \frac{\rho_{f}^{( \pm \ell)}(n)}{n} \exp (-n / x) .
$$

On a l'expression intégrale

$$
\omega_{f}^{( \pm \ell)}(x)=\frac{1}{2 \pi i} \int_{(1)} L\left(s+1, \operatorname{sym}^{2} f\right)^{ \pm \ell} \Gamma(s) x^{s} \mathrm{~d} s .
$$

Pour tout $\varepsilon>0$, il existe alors un réel $\iota(\varepsilon)>0$ tel que pour tous $\ell \in \mathbb{N}^{*}$, $x \geq 1, N$ sans facteur carré et toute forme $f \in \mathrm{H}_{k}^{*}(N)$, on a la majoration

$$
\left|\omega_{f}^{( \pm \ell)}(x)\right| \leq \iota(\varepsilon)^{\ell} x^{\varepsilon}
$$

Cela résulte du déplacement, dans (4.57) de la droite (1) vers la droite $(\varepsilon)$ puis de (3.22) et de la majoration de Stirling.

On veut remplacer $L\left(1, \mathrm{sym}^{2} f\right)^{ \pm \ell}$ par la somme courte (4.56), on estime l'erreur commise dans le

Lemme 22. Soient $N$ un entier sans facteur carré, $\ell \in \mathbb{N}^{*}$ et $x \geq 1$. Pour $0<\eta \leq \frac{1}{22}$ et $f \in \mathrm{H}_{k}^{+}(N ; \eta)$, on a

$$
L\left(1, \operatorname{sym}^{2} f\right)^{ \pm \ell}=\omega_{f}^{( \pm \ell)}(x)+O\left(R_{1}^{( \pm \ell)}\right)
$$

où

$$
R_{1}^{( \pm \ell)}:=e^{\ell D_{ \pm}(\eta)[\log (2 N)]^{\theta(\eta)}}\left\{x^{-\eta} \frac{[\log (2 N)]^{2}}{\eta}+x e^{-\pi[\log (2 N)]^{2} / 3}\right\}
$$

puis $D_{ \pm}(\eta)$ et $\theta(\eta)$ sont définis comme dans les lemmes 9 et 11. La constante impliquée dans le symbole $O$ est absolue. 
Démonstration. Soit $\mathcal{C}$ le chemin reliant $1-i \infty, 1-i[\log (2 N)]^{2},-\eta-$ $i[\log (2 N)]^{2},-\eta+i[\log (2 N)]^{2}, 1+i[\log (2 N)]^{2}$ et $1+i \infty$. Le théorème de résidus implique que

$$
\omega_{f}^{( \pm \ell)}(x)=L\left(1, \operatorname{sym}^{2} f\right)^{ \pm \ell}+\frac{1}{2 \pi i} \int_{\mathcal{C}} L\left(s+1, \operatorname{sym}^{2} f\right)^{ \pm \ell} \Gamma(s) x^{s} \mathrm{~d} s .
$$

D'après les lemmes 9 et 11 et l'équation (3.22), on a, pour $s \in \mathcal{C}$,

$$
\left|L\left(s+1, \operatorname{sym}^{2} f\right)\right|^{ \pm \ell} \leq e^{\ell D_{ \pm}(\eta)[\log (2 N)]^{\theta(\eta)}} .
$$

Puisque $s \Gamma(s)=\Gamma(s+1)$, on déduit, pour $s=-\eta+i \tau$, que

$$
\eta|\Gamma(s)| \leq|\Gamma(1-\eta+i \tau)| \leq \Gamma(1-\eta)=\frac{\Gamma(2-\eta)}{1-\eta} \leq \frac{1}{1-\eta},
$$

d'où

$$
|\Gamma(s)| \leq \frac{22}{21 \eta} \quad(s=-\eta+i \tau) .
$$

L'estimation de Stirling implique, pour $-\eta \leq \sigma \leq 1$ et $|\tau| \geq[\log (2 N)]^{2}$, que

$$
|\Gamma(s)| \leq C e^{-\pi|\tau| / 2}|\tau|^{1 / 2} .
$$

En utilisant (4.59), (4.60) et (4.61) on obtient

$$
\begin{aligned}
& \mid \int_{\mathcal{C}} L(s\left.+1, \operatorname{sym}^{2} f\right)^{ \pm \ell} \Gamma(s) x^{s} \mathrm{~d} s\left|\leq e^{\ell D_{ \pm}(\eta)[\log (2 N)]^{\theta(\eta)}} \int_{\mathcal{C}} x^{\sigma}\right| \Gamma(s) \mathrm{d} s \mid \\
& \ll e^{\ell D_{ \pm}(\eta)[\log (2 N)]^{\theta(\eta)}}\left\{x^{-\eta} \frac{[\log (2 N)]^{2}}{\eta}+x e^{-\pi[\log (2 N)]^{2} / 2} \log (2 N)\right\}
\end{aligned}
$$

où la constante impliquée dans « est absolue. Cela achève la démonstration.

On peut alors calculer la moyenne de (4.56) dans le

Lemme 23. Si $N$ est un entier sans facteur carré, alors pour tout $x \geq 1$ et tout $\ell \in \mathbb{N}^{*}$ on a

où

$$
\sum_{f \in \mathrm{H}_{k}^{*}(N)} \omega(f) \omega_{f}^{( \pm \ell)}(x)=\frac{\varphi(N)}{N} M_{ \pm \ell}^{(N)}+O_{k}\left(R_{2}\right)
$$

et

$$
M_{ \pm \ell}^{(N)}:=\sum_{n=1}^{+\infty} \frac{m_{ \pm \ell}^{(N)}(n)}{n}
$$

$R_{2}:=\frac{\tau(N)^{2}}{N}[\log (2 N)] x^{1 / 2}\left\{(C \ell)^{C \ell}+\left[\log ^{C}(3 \ell) \log (3 x)\right]^{3 \ell}\right\}+x^{-1 / 2+\varepsilon} e^{\iota(\varepsilon) \ell^{2}}$, avec $\iota(\varepsilon)$ une constante ne dépendant que de $\varepsilon$. La constante impliquée dans le symbole $O_{k}$ ne dépend que de $k$. 
Démonstration. D'après (4.56), le lemme 12 et le lemme 17, on peut écrire

$$
\begin{aligned}
& \sum_{f \in \mathrm{H}_{k}^{*}(N)} \omega(f) \omega_{f}^{( \pm \ell)}(x) \\
& =\frac{\varphi(N)}{N} \sum_{n \geq 1} \frac{m_{ \pm \ell}^{(N)}(n)}{n} \exp (-n / x)+O_{k}\left(\frac{\tau(N)^{2}}{N} \log (2 N) R_{3}^{( \pm \ell)}\right)
\end{aligned}
$$

où

$$
R_{3}^{( \pm \ell)}:=\sum_{n \geq 1} r_{ \pm \ell}(n) n^{-1 / 2} \log (2 n) \exp (-n / x)
$$

En utilisant les lemmes 16 et 21, une intégration par parties conduit à

$$
R_{3}^{( \pm \ell)} \leq[\log (3 \ell)]^{C \ell} \int_{1}^{\infty} t^{-1 / 2} e^{-t / x}[\log (3 t)]^{3 \ell}\left(1+\frac{t}{x}\right) \mathrm{d} t
$$

Mais

$$
\int_{1}^{x} t^{-1 / 2} e^{-t / x}[\log (3 t)]^{3 \ell}\left(1+\frac{t}{x}\right) \mathrm{d} t \ll x^{1 / 2}[\log (3 x)]^{3 \ell}
$$

et

$$
\begin{aligned}
\int_{x}^{\infty} t^{-1 / 2} e^{-t / x}[\log (3 t)]^{3 \ell}\left(1+\frac{t}{x}\right) \mathrm{d} t & \ll x^{1 / 2} \int_{1}^{\infty} u^{1 / 2} e^{-u}[\log (3 u x)]^{3 \ell} \mathrm{d} u \\
& \ll x^{1 / 2}\left\{(C \ell)^{C \ell}+[2 \log (3 x)]^{3 \ell}\right\}
\end{aligned}
$$

d'où

$$
\begin{aligned}
R_{3}^{( \pm \ell)} & \ll x^{1 / 2}[\log (3 \ell)]^{C \ell}\left\{(C \ell)^{C \ell}+[2 \log (3 x)]^{3 \ell}\right\} \\
& \ll x^{1 / 2}\left\{(C \ell)^{C \ell}+\left[\log ^{C}(3 \ell) \log (3 x)\right]^{3 \ell}\right\} .
\end{aligned}
$$

Compte-tenu des lemmes 14 et 19, on a [22, page 151]

$\sum_{n \geq 1} \frac{m_{ \pm \ell}^{(N)}(n)}{n} \exp (-n / x)=\frac{1}{2 \pi i} \int_{(1)} \zeta^{(N)}(2 s+2)^{\ell(\ell \pm 1) / 2} \mathcal{M}_{ \pm \ell}^{(N)}(s+1) \Gamma(s) x^{s} \mathrm{~d} s$

En déplaçant la ligne d'intégration $(1)$ à $(-1 / 2+\varepsilon)$, le théorème des résidus implique

$$
\sum_{n \geq 1} \frac{m_{ \pm \ell}^{(N)}(n)}{n} \exp (-n / x)=\sum_{n \geq 1} \frac{m_{ \pm \ell}^{(N)}(n)}{n}+R_{4}^{( \pm \ell)}
$$


où

$$
\begin{aligned}
R_{4}^{( \pm \ell)} & :=\frac{1}{2 \pi i} \int_{(-1 / 2+\varepsilon)} \zeta^{(N)}(2 s+2)^{\ell(\ell \pm 1) / 2} \mathcal{M}_{ \pm \ell}^{(N)}(s+1) \Gamma(s) x^{s} \mathrm{~d} s \\
& \ll_{\varepsilon} x^{-1 / 2+\varepsilon} \varepsilon^{-\ell(\ell \pm 1) / 2} e^{\iota(\varepsilon) \ell^{2}} \int_{(-1 / 2+\varepsilon)}|\Gamma(s)| \mathrm{d} \tau \\
& \ll_{\varepsilon} x^{-1 / 2+\varepsilon} e^{\ell(\varepsilon) \ell^{2}}
\end{aligned}
$$

grâce à l'estimation de Stirling et aux lemmes 14 et 19 .

Posant

$$
R_{2}^{( \pm \ell)}:=\frac{\tau(N)^{2}}{N} \log (2 N) R_{3}^{( \pm \ell)}+\frac{\varphi(N)}{N} R_{4}^{( \pm \ell)}
$$

on déduit de (4.62) et (4.64) que

$$
\sum_{f \in \mathrm{H}_{k}^{*}(N)} \omega(f) \omega_{f}^{( \pm \ell)}(x)=\frac{\varphi(N)}{N} M_{ \pm \ell}^{(N)}+O\left(R_{2}^{( \pm \ell)}\right) .
$$

La majoration $R_{2}^{( \pm \ell)} \ll R_{2}$ résulte alors de (4.63) et (4.65).

On complète alors la démonstration du théorème B. En utilisant les majorations $(3.17),(3.19)$ et $(1.1)$, on voit que

$$
\sum_{f \in \mathrm{H}_{k}^{-}(N ; \eta)} \omega(f) L\left(1, \operatorname{sym}^{2} f\right)^{ \pm \ell} \leq \xi N^{19 \eta-1}[\log (2 N)]^{\ell+\xi} .
$$

Le lemme 22 et les majorations (4.66) et (3.9) donnent

$$
\sum_{f \in \mathrm{H}_{k}^{*}(N)} \omega(f) L\left(1, \operatorname{sym}^{2} f\right)^{ \pm \ell}
$$

$$
\begin{aligned}
& =\sum_{f \in \mathrm{H}_{k}^{+}(N ; \eta)} \omega(f) L\left(1, \operatorname{sym}^{2} f\right)^{ \pm \ell}+O_{k}\left(N^{19 \eta-1}[\log (2 N)]^{\ell+\xi}\right) \\
& =\sum_{f \in \mathrm{H}_{k}^{+}(N ; \eta)} \omega(f) \omega_{f}^{( \pm \ell)}(x)+O_{k}\left(R_{5}^{( \pm \ell)}\right)
\end{aligned}
$$

où

$$
\begin{aligned}
R_{5}^{( \pm \ell)}:=N^{19 \eta-1} & {[\log (2 N)]^{\ell+\xi}+} \\
& +e^{\ell D_{ \pm}(\eta)[\log (2 N)]^{\theta(\eta)}}\left\{x^{-\eta} \frac{[\log (2 N)]^{2}}{\eta}+x e^{-\pi[\log (2 N)]^{2} / 3}\right\}
\end{aligned}
$$

et la constante impliquée dans le symbole $O_{k}$ ne dépend que de $k$.

Il résulte de (4.58), (3.19) et (3.17) que

$$
\left|\sum_{f \in \mathrm{H}_{k}^{-}(N ; \eta)} \omega(f) \omega_{f}^{( \pm \ell)}(x)\right| \leq \xi \iota(\varepsilon)^{\ell} x^{\varepsilon} N^{19 \eta-1}[\log (2 N)]^{\xi} .
$$


Grâce à (4.68), on peut réintroduire les formes de $\mathrm{H}_{k}^{-}(N ; \eta)$ dans $(4.67)$ et obtenir

$$
\sum_{f \in \mathrm{H}_{k}^{*}(N)} \omega(f) L\left(1, \operatorname{sym}^{2} f\right)^{ \pm \ell}=\sum_{f \in \mathrm{H}_{k}^{*}(N)} \omega(f) \omega_{f}^{( \pm \ell)}(x)+O_{k}\left(R_{6}^{( \pm \ell)}\right),
$$

où

$$
R_{6}^{( \pm \ell)}:=R_{5}^{( \pm \ell)}+\iota(\varepsilon)^{\ell} x^{\varepsilon} N^{19 \eta-1}[\log (2 N)]^{\xi}
$$

et la constante impliquée dans le symbole $O_{k}$ ne dépend que de $k$.

Le lemme 23 implique alors

$$
\sum_{f \in \mathrm{H}_{k}^{*}(N)} \omega(f) L\left(1, \mathrm{sym}^{2} f\right)^{ \pm \ell}=\frac{\varphi(N)}{N} M_{ \pm \ell}^{(N)}+O_{k}\left(R_{7}^{( \pm \ell)}\right)
$$

avec

$$
\begin{aligned}
R_{7}^{( \pm \ell)}= & \frac{\tau(N)^{2}}{N}[\log (2 N)] x^{1 / 2}\left\{(C \ell)^{C \ell}+\left[\log ^{C}(3 \ell) \log (3 x)\right]^{3 \ell}\right\} \\
& +x^{-1 / 2+\varepsilon} e^{\iota(\varepsilon) \ell^{2}} \\
& +e^{\ell D_{ \pm}(\eta)[\log (2 N)]^{\theta(\eta)}}\left\{x^{-\eta} \frac{[\log (2 N)]^{2}}{\eta}+x e^{-\pi[\log (2 N)]^{2} / 3}\right\} \\
& +N^{19 \eta-1}[\log (2 N)]^{\ell+\xi}+\iota(\varepsilon)^{\ell} x^{\varepsilon} N^{19 \eta-1}[\log (2 N)]^{\xi}
\end{aligned}
$$

où la constante impliquée dans le symbole $O_{k}$ ne dépend que de $k$.

En prenant $\eta=1 / 22, \varepsilon=7 / 242$ et $x=N^{11 / 6}$, on obtient

$$
\begin{aligned}
R_{7}^{( \pm \ell)} \ll & N^{-1 / 12} \tau(N)^{2} \log (2 N)\left\{(C \ell)^{C \ell}+\left[\log ^{C}(3 \ell) \log (2 N)\right]^{3 \ell}\right\} \\
& +N^{-19 / 22} e^{C \ell^{2}} \\
& +e^{\xi \ell[\log (2 N)]^{\theta(1 / 22)}}\left\{N^{-1 / 12}[\log (2 N)]^{2}+N^{11 / 6} e^{-\pi[\log (2 N)]^{2} / 3}\right\} \\
& +N^{-3 / 22}[\log (2 N)]^{\ell+\xi}+N^{-1 / 12}[\log (2 N)]^{\xi} \xi^{\ell} .
\end{aligned}
$$

Puisque $\theta\left(\frac{1}{22}\right) \leq \frac{1}{2}$, on a

$$
R_{7}^{( \pm \ell)} \ll N^{-1 / 13} e^{\xi \ell[\log (2 N)]^{1 / 2}+C \ell^{2}}
$$

Le report de (4.70) dans (4.69) conduit au théorème B. 


\subsection{Preuve du corollaire A}

Dans cette partie, on montre l'existence de moments de $f \mapsto L\left(1, \operatorname{sym}^{2} f\right)$ lorsque le niveau tend vers $+\infty$ dans $\mathcal{N}_{\text {cri }}$.

Puisque $m_{ \pm \ell}^{(N)}(n)$ est multiplicative, les égalités (4.15), (4.17) et (4.34) et (4.37) donnent

$$
\frac{\varphi(N)}{N} M_{ \pm \ell}^{(N)}=M_{ \pm \ell} \prod_{p \mid N}\left[\left(1-\frac{1}{p}\right)\left(1-\frac{1}{p^{2}}\right)^{\mp \ell}\left(1+\sum_{\nu \geq 2} \frac{m_{ \pm \ell, \nu}}{p^{\nu}}\right)^{-1}\right]
$$

avec

$$
M_{ \pm \ell}:=\prod_{p \in \mathcal{P}} \sum_{\nu=0}^{+\infty} \frac{m_{ \pm \ell, \nu}}{p^{\nu}}
$$

Grâce à (4.36) et (4.35), on peut récrire, pour $\ell \in \mathbb{N}^{*}$,

$$
M_{-\ell}=\prod_{p \in \mathcal{P}} \sum_{\nu=0}^{+\infty} \frac{\widehat{m}_{-\ell, \nu}}{p^{\nu}}
$$

et

$$
M_{+\ell}=\zeta(2)^{\ell} \prod_{p \in \mathcal{P}} \sum_{\nu=0}^{+\infty} \frac{\widehat{m}_{+\ell, \nu}}{p^{\nu}} .
$$

Par (1.8) et (1.7), on déduit du théorème $B$ le résultat du corollaire A lorsque $\ell>\frac{1}{2} \sqrt{\log (2 N)}$. On peut donc supposer que $\ell \leq \frac{1}{2} \sqrt{\log (2 N)}$. Puisque $P^{-}(N) \geq \log (2 N)$, on a $p \geq 4 \ell^{2}$ pour $p \mid N$. Dans le cas des moments positifs, l'égalité (4.17) donne pour $p \mid N$,

$$
\left(1-\frac{1}{p^{2}}\right)^{-\ell}\left(1+\sum_{\nu \geq 2} \frac{m_{+\ell, \nu}}{p^{\nu}}\right)^{-1}=\left(1+\sum_{\nu \geq 2} \frac{\widehat{m}_{+\ell, \nu}}{p^{\nu}}\right)^{-1} .
$$

En utilisant $(4.74),(4.25)$ avec $p$ à la place $p^{1 / 2}$ et $\widehat{m}_{+\ell, 2}=\ell(\ell-1) / 2$, on obtient, pour $p \mid N$,

$$
\left(1-\frac{1}{p^{2}}\right)^{-\ell}\left(1+\sum_{\nu \geq 2} \frac{m_{+\ell, \nu}}{p^{\nu}}\right)^{-1}=1+O\left(\frac{\ell^{2}}{p^{2}}\right)=1+O\left(\frac{\log (2 N)}{P^{-}(N)^{2}}\right) .
$$

Dans le cas des moments négatifs, (4.36), (4.45) avec $p$ à la place de $p^{1 / 2}$ et $\widehat{m}_{-\ell, 2}=\ell(\ell-1) / 2$ impliquent, pour $p \mid N$,

$$
\begin{aligned}
\left(1-\frac{1}{p^{2}}\right)^{\ell}\left(1+\sum_{\nu \geq 2} \frac{m_{-\ell, \nu}}{p^{\nu}}\right)^{-1} & =\left\{1+O\left(\frac{\ell}{p^{2}}\right)\right\}\left\{1+O\left(\frac{\ell^{2}}{p^{2}}\right)\right\} \\
& =1+O\left(\frac{\log (2 N)}{P^{-}(N)^{2}}\right) .
\end{aligned}
$$


En désignant par $\omega(N)$ le nombre de facteurs premiers distincts de $N$, les deux estimations précédentes nous permettent d'écrire

$$
\begin{aligned}
& \prod_{p \mid N}\left\{\left(1-\frac{1}{p}\right)\left(1-\frac{1}{p^{2}}\right)^{\mp \ell}\left(1+\sum_{\nu \geq 2} \frac{m_{ \pm \ell, \nu}}{p^{\nu}}\right)^{-1}\right\} \\
& =1+O\left(\frac{\omega(N)}{P^{-}(N)}\left[1+\frac{\log (2 N)}{P^{-}(N)}\right]\right)=1+O\left(\frac{1}{\log _{2}(3 N)}\right)
\end{aligned}
$$

$\operatorname{car} \omega(N) \ll \log (2 N) / \log _{2}(3 N)$ et $P^{-}(N) \geq \log (2 N)$.

Le report de (4.75) dans (4.71) donne alors le résultat pour $\ell \leq \frac{1}{2} \sqrt{\log (2 N)}$.

\subsection{Preuve du corollaire B}

Pour la limite inférieure, on a

$$
M_{ \pm \ell}^{\left(N_{j}\right)}=\left[\zeta(2) \zeta^{\left(N_{j}\right)}(2)^{-1}\right]^{ \pm \ell} \prod_{p>p_{j}} \sum_{\nu \geq 0} \frac{m_{ \pm \ell, \nu}}{p^{\nu}}
$$

de sorte que la convergence des produits (1.5) conduit, $\ell$ étant fixé, à

$$
\lim _{j \rightarrow+\infty} M_{ \pm \ell}^{\left(N_{j}\right)}=\zeta(2)^{ \pm \ell}
$$

Puisqu'il existe $C>0$ tel que

$$
\frac{\varphi\left(N_{j}\right)}{N_{j}} \sim \frac{C}{\log _{2}\left(N_{j}\right)}, \quad j \rightarrow+\infty
$$

on en déduit

$$
\lim _{j \rightarrow \infty} \frac{\varphi\left(N_{j}\right)}{N_{j}} M_{ \pm \ell}^{\left(N_{j}\right)}=0 .
$$

On conclut en remarquant que la limite inférieure recherchée est positive.

On étudie ensuite la limite supérieure. Grâce au corollaire A, on a immédiatement

$$
\limsup _{\substack{N \rightarrow \infty \\ \mu(N) \neq 0}} \frac{\varphi(N)}{N} M_{ \pm \ell}^{(N)} \geq M_{ \pm \ell}
$$

Pour les moments positifs, grâce à (4.17) on a

$$
\frac{M_{+\ell}}{M_{+\ell}^{(N)}}=\prod_{p \mid N}\left[\left(1-\frac{1}{p^{2}}\right)^{\ell} \sum_{\nu=0}^{+\infty} \frac{m_{+\ell, \nu}}{p^{\nu}}\right] .
$$


Pour $p \mid N$, l'égalité (4.17) donne aussi

$$
\sum_{\nu=0}^{+\infty} \frac{m_{+\ell, \nu}}{p^{\nu}}=\left(1-\frac{1}{p^{2}}\right)^{-\ell} \sum_{\nu=0}^{+\infty} \frac{\widehat{m}_{+\ell, \nu}}{p^{\nu}}
$$

d'où

$$
\frac{M_{+\ell}}{M_{+\ell}^{(N)}}=\prod_{p \mid N} \sum_{\nu=0}^{+\infty} \frac{\widehat{m}_{+\ell, \nu}}{p^{\nu}} \geq 1
$$

On en déduit

$$
\limsup _{\substack{N \rightarrow \infty \\ \mu(N) \neq 0}} \frac{\varphi(N)}{N} M_{+\ell}^{(N)} \leq M_{+\ell} .
$$

Par (4.77), (4.78) et (1.8) on a donc

$$
\limsup _{\substack{N \rightarrow \infty \\ \mu(N) \neq 0}} \frac{\varphi(N)}{N} M_{+\ell}^{(N)}=M_{+\ell}=e^{3 \ell \log \log \ell+O(\ell)} .
$$

Pour les moments négatifs, les équations (4.77) et (1.7) donnent

$$
\limsup _{\substack{N \rightarrow \infty \\ \mu(N) \neq 0}} \frac{\varphi(N)}{N} M_{-\ell}^{(N)} \geq e^{\ell \log \log \ell+O(\ell)} .
$$

Pour prouver l'égalité dans cette équation, il suffit donc, toujours grâce à (1.7), de prouver l'existence de $C>0$ telle que, pour tout $\ell>0$ on ait

$$
M_{-\ell}^{(N)} \leq M_{-\ell} e^{C \ell}
$$

Pour prouver (4.79), on utilise le $\S 2.4$ de [19] en en conservant toutes les notations sauf $\ell_{n}$ qui devient $\mathcal{L}_{n}$. Compte-tenu de [19, proposition 11] et (1.6), on a

$$
M_{-\ell}=\frac{1}{\zeta(3)^{\ell}} \prod_{p} \mathcal{L}_{\ell}\left(\frac{p}{p^{2}+p+1}\right) .
$$

D'après (4.71) on a

$$
M_{-\ell}^{(N)} \leq M_{-\ell} \prod_{p \mid N}\left(\sum_{\nu \geq 0} \frac{m_{-\ell, \nu}}{p^{\nu}}\right)^{-1} .
$$

Or, comme dans la preuve de [19, proposition 11], on a

$$
\prod_{p \mid N}\left(\sum_{\nu \geq 0} \frac{m_{-\ell, \nu}}{p^{\nu}}\right)=\prod_{p \mid N}\left(1-\frac{1}{p^{3}}\right)^{\ell} \prod_{p \mid N} \mathcal{L}_{\ell}\left(x_{p}\right)
$$

avec $x_{p}:=p /\left(p^{2}+p+1\right)$. 
On prouve dans $[19, \S 2.4]$ l'inégalité

$$
\frac{\mathcal{L}_{\ell}\left(x_{p}\right)}{\left(1+x_{p}\right)^{\ell}} \geq \frac{1}{\pi \sqrt{2}}\left[1-\frac{1}{2(\ell+1)}\right]^{\ell}\left[\frac{1+x_{p}}{(\ell+1) x_{p}}\right]^{1 / 2} .
$$

On en déduit l'existence d'une constante $\left.C_{0} \in\right] 0,1[$ telle que

$$
\frac{\mathcal{L}_{\ell}\left(x_{p}\right)}{\left(1+x_{p}\right)^{\ell}} \geq C_{0}\left[\frac{1+x_{p}}{(\ell+1) x_{p}}\right]^{1 / 2} \text {. }
$$

Pour $\ell x_{p}>1$, on a $(\ell+1) x_{p}>1+x_{p}$ de sorte que

$$
\begin{aligned}
\log \prod_{\substack{p \mid N \\
\ell x_{p}>1}}\left[\frac{1+x_{p}}{(\ell+1) x_{p}}\right]^{1 / 2} & \geq-\frac{1}{2} \sum_{p \leq \ell} \log \frac{(\ell+1) x_{p}}{1+x_{p}} \\
& \geq-C_{1} \frac{\ell}{\log \ell}
\end{aligned}
$$

avec $C_{1}>0$ une constante grâce au théorème des nombres premiers. On déduit alors de (4.82) et (4.83) l'existence d'une constante $C_{2}>0$ telle que

$$
\begin{aligned}
\log \prod_{\substack{p \mid N \\
\ell x_{p}>1}} \mathcal{L}_{\ell}\left(x_{p}\right) & \geq \log \prod_{\substack{p \mid N \\
\ell x_{p}>1}} \frac{\mathcal{L}_{\ell}\left(x_{p}\right)}{\left(1+x_{p}\right)^{\ell}} \\
& \geq-C_{2} \frac{\ell}{\log \ell} .
\end{aligned}
$$

Toujours d'après [19, §2.4], pour $\ell x_{p} \leq 1$, on a

$$
\mathcal{L}_{\ell}\left(x_{p}\right)=1+O\left(\left(\ell x_{p}\right)^{2}\right)
$$

d'où l'on déduit l'existence d'une constante $C_{3}>0$ telle que

$$
\log \prod_{\substack{p \mid N \\ \ell x_{p} \leq 1}} \mathcal{L}_{\ell}\left(x_{p}\right) \geq-C_{3} \frac{\ell}{\log \ell} .
$$

De (4.84) et (4.85) on déduit l'existence d'une constante $C_{4}>0$ telle que

$$
\prod_{p \mid N} \mathcal{L}_{\ell}\left(x_{p}\right) \geq \exp \left(-C_{4} \frac{\ell}{\log \ell}\right) .
$$

D'autre part, il existe une constante $C_{5}>0$ telle que

$$
\prod_{p \mid N}\left(1-\frac{1}{p^{3}}\right)^{\ell} \geq \exp \left(-C_{5} \ell\right) .
$$

Le report de (4.86) et (4.87) dans (4.81), puis du résultat dans (4.80) conduit à (4.79) ce qui achève la preuve. 


\section{Preuve du théorème $A$}

On se place dans le cadre des hypothèses du théorème $\mathrm{A}$. On prouve la minoration de $\mathrm{H}_{k}^{*+}\left(N ; C, \mathrm{sym}^{2}\right)$. Celle de $\mathrm{H}_{k}^{*-}\left(N ; C\right.$, sym$\left.{ }^{2}\right)$ se démontre de la même façon en remplaçant (1.8) par (1.7). L'existence de $f_{ \pm}$en résulte puisque

$$
\lim _{N \rightarrow \infty} e^{-\xi[\log (3 N)]^{\varepsilon}} \# \mathrm{H}_{k}^{*}(N)=+\infty .
$$

Soit $\alpha: \mathcal{N}_{\text {cri }} \rightarrow \mathbb{R}^{+}$une application. Par l'inégalité de Cauchy-Schwarz, on a

$$
\sum_{\substack{f \in \mathrm{H}_{k}^{*}(N) \\ L\left(1, \mathrm{sym}^{2} f\right) \geq \alpha(N)}} L\left(1, \operatorname{sym}^{2} f\right)^{\ell} \leq X_{\alpha}(N)^{1 / 2}\left[\sum_{f \in \mathrm{H}_{k}^{*}(N)} L\left(1, \operatorname{sym}^{2} f\right)^{2 \ell}\right]^{1 / 2}
$$

avec

$$
X_{\alpha}(N):=\#\left\{f \in \mathrm{H}_{k}^{*}(N): L\left(1, \operatorname{sym}^{2} f\right) \geq \alpha(N)\right\} .
$$

D'autre part,

$$
\begin{aligned}
\sum_{\substack{f \in \mathrm{H}_{k}^{*}(N) \\
L\left(1, \mathrm{sym}^{2} f\right) \geq \alpha(N)}} L\left(1, \operatorname{sym}^{2} f\right)^{\ell} & =\sum_{f \in \mathrm{H}_{k}^{*}(N)} L\left(1, \operatorname{sym}^{2} f\right)^{\ell}-\sum_{\substack{f \in \mathrm{H}_{k}^{*}(N) \\
L\left(1, \mathrm{sym}^{2} f\right)<\alpha(N)}} L\left(1, \operatorname{sym}^{2} f\right)^{\ell} \\
& \geq \sum_{f \in \mathrm{H}_{k}^{*}(N)} L\left(1, \operatorname{sym}^{2} f\right)^{\ell}-\alpha(N)^{\ell} \# \mathrm{H}_{k}^{*}(N) .
\end{aligned}
$$

On déduit de (5.1), (5.2) et (3.18) que

$$
X_{\alpha}(N) \geq P_{\alpha}(N, \ell) \# \mathrm{H}_{k}^{*}(N)
$$

avec

$$
P_{\alpha}(N, \ell)=\frac{\left[\frac{(k-1) N}{2 \pi^{2} \# \mathrm{H}_{k}^{*}(N)} \sum_{f \in \mathrm{H}_{k}^{*}(N)} \omega(f) L\left(1, \operatorname{sym}^{2} f\right)^{\ell+1}-\alpha(N)^{\ell}\right]^{2}}{\frac{(k-1) N}{2 \pi^{2} \# \mathrm{H}_{k}^{*}(N)} \sum_{f \in \mathrm{H}_{k}^{*}(N)} \omega(f) L\left(1, \operatorname{sym}^{2} f\right)^{2 \ell+1}}
$$

tant que ce qui est à l'intérieur du carré est positif. Compte-tenu de (3.4) et du corollaire $(\mathrm{A})$, le choix de

$$
\alpha(N)=\left[\frac{N M_{+(\ell+1)}}{2 \varphi(N)}\right]^{1 / \ell}
$$


conduit à l'existence d'une constante $K>0$ telle que

$$
P_{\alpha}(N, \ell) \geq K \frac{M_{+(\ell+1)}^{2}}{M_{+(2 \ell+1)}}
$$

Compte-tenu de (1.8), on a

$$
\frac{M_{+(\ell+1)}^{2}}{M_{+(2 \ell+1)}} \geq \exp \left(-\xi \frac{\ell}{\log \ell}\right) .
$$

Le choix de $\ell=\left\lfloor\{\log (3 N)\}^{\varepsilon}\right\rfloor$ et la minoration $\alpha(N) \geq C\left[\log _{2}(3 N)\right]^{3}$ conduisent à la minoration recherchée.

\section{Preuve du théorème $\mathrm{C}$}

D'après (3.18), on peut écrire

$$
\sum_{f \in \mathrm{H}_{k}^{*}(N)} L\left(1, \operatorname{sym}^{2} f\right)=\frac{k-1}{2 \pi^{2}} N \sum_{f \in \mathrm{H}_{k}^{*}(N)} \omega(f) L\left(1, \operatorname{sym}^{2} f\right)^{2} .
$$

Dans (4.57), en déplaçant la ligne d'intégration (1) à $\left(-\frac{1}{2}\right)$, le théorème des résidus implique

$$
L\left(1, \operatorname{sym}^{2} f\right)^{2}=\omega_{f}^{(+2)}(x)-R(f, x)
$$

où

$$
R(f, x):=\frac{1}{2 \pi i} \int_{(-1 / 2)} L\left(s+1, \operatorname{sym}^{2} f\right)^{2} \Gamma(s) x^{s} \mathrm{~d} s .
$$

En reportant (6.2) dans (6.1), on obtient

$$
\sum_{f \in \mathrm{H}_{k}^{*}(N)} L\left(1, \operatorname{sym}^{2} f\right)=\frac{k-1}{2 \pi^{2}} N \sum_{f \in \mathrm{H}_{k}^{*}(N)} \omega(f) \omega_{f}^{(+2)}(x)+O_{k}\left(N\left|R^{\prime}\right|\right),
$$

où

$$
R^{\prime}:=\int_{(-1 / 2)} \sum_{f \in \mathrm{H}_{k}^{*}(N)} \omega(f) L\left(s+1, \operatorname{sym}^{2} f\right)^{2} \Gamma(s) x^{s} \mathrm{~d} s .
$$

De (3.19) on déduit

$$
R^{\prime} \ll x^{-1 / 2} N^{-1}[\log (2 N)] \int_{(-1 / 2)} \sum_{f \in \mathrm{H}_{k}^{*}(N)}\left|L\left(s+1, \operatorname{sym}^{2} f\right)\right|^{2}|\Gamma(s)| \mathrm{d} \tau .
$$


Maintenant le lemme 7 permet de déduire

$$
R^{\prime} \ll N^{\varepsilon} x^{-1 / 2} \int_{(-1 / 2)}\left|s^{8} \Gamma(s)\right| \mathrm{d} \tau \ll N^{\varepsilon} x^{-1 / 2} .
$$

Donc on obtient

(6.3) $\sum_{f \in \mathrm{H}_{k}^{*}(N)} L\left(1, \operatorname{sym}^{2} f\right)=\frac{k-1}{2 \pi^{2}} N \sum_{f \in \mathrm{H}_{k}^{*}(N)} \omega(f) \omega_{f}^{(+2)}(x)+O_{\varepsilon, k}\left(N^{1+\varepsilon} x^{-1 / 2}\right)$.

En utilisant le lemme 23 avec $\ell=2$, on a

$$
\sum_{f \in \mathrm{H}_{k}^{*}(N)} \omega(f) \omega_{f}^{(+2)}(x)=\frac{\varphi(N)}{N} M_{+2}^{(N)}+O_{\varepsilon, k}\left(N^{-1+\varepsilon} x^{1 / 2+\varepsilon}+x^{-1 / 2+\varepsilon}\right) .
$$

De (4.16) et (4.17), on déduit que

$$
m_{+2}^{(N)}=\mu_{N}^{+} * \mu_{N}^{+} * \square^{(N)} * \square^{(N)} * \square^{(N)},
$$

d'où

$$
M_{+2}^{(N)}=\zeta(2)^{3} \prod_{p \mid N}\left(1-\frac{1}{p^{2}}\right) .
$$

L'équation (6.4) devient donc

$$
\begin{aligned}
\frac{k-1}{2 \pi^{2}} N \sum_{f \in \mathrm{H}_{k}^{*}(N)} \omega & (f) \omega_{f}^{(+2)}(x) \\
& =\frac{\pi^{4}}{432}(k-1) \psi(N)+O_{\varepsilon, k}\left(N^{\varepsilon} x^{1 / 2+\varepsilon}+N x^{-1 / 2+\varepsilon}\right)
\end{aligned}
$$

avec

$$
\psi(N):=N \prod_{p \mid N}\left(1-\frac{1}{p}\right)\left(1-\frac{1}{p^{2}}\right) .
$$

En reportant (6.5) dans (6.3), on trouve

(6.6) $\sum_{f \in \mathrm{H}_{k}^{*}(N)} L\left(1, \operatorname{sym}^{2} f\right)=\frac{\pi^{4}}{432}(k-1) \psi(N)+O_{\varepsilon, k}\left(N^{\varepsilon} x^{1 / 2+\varepsilon}+N x^{-1 / 2+\varepsilon}\right)$.

Le théorème $\mathrm{C}$ résulte de (6.6) en prenant $x=N$. 


\section{Références}

[1] Akbary, A. : Average values of symmetric square $L$-functions at $\operatorname{Re}(s)=2$. C. R. Math. Acad. Sci. Soc. R. Can. 22 (2000), no. 3, 97-104.

[2] Duke, W. And Kowalski, E. : A problem of Linnik for elliptic curves and mean-value estimates for automorphic representations. Invent. Math. 139 (2000), no. 1, 1-39.

[3] Duke, W. : Extreme values of Artin $L$-functions and class numbers. Compositio Math. 136 (2003), no. 1, 103-115.

[4] Frey, G. : On ternary equations of Fermat type and relations with elliptic curves. In Modular forms and Fermat's last theorem (Boston, MA, 1995), 527-548. Springer, New York, 1997.

[5] Goldfeld, D., Hoffstein, J. and Lieman, D. : An effective zero-free region. Ann. of Math. (2) 140 (1994), no. 1, 177-181, appendice de [7].

[6] Habsieger, L. ANd Royer, E. : Interprétation combinatoire des moments positifs des valeurs de fonctions $L$ au bord de la bande critique. À paraître dans Ann. Inst. Fourier (Grenoble).

[7] Hoffitein, J. and Lockhart, P. : Coefficients of Maass forms and the Siegel zero. Ann. of Math. (2) 140 (1994), no. 1, 161-181.

[8] Iwaniec, H., Luo, W. and Sarnak, P. : Low lying zeros of families of L-functions. Inst. Hautes Études Sci. Publ. Math. No. 91 (2000), 55-131 (2001).

[9] Inaniec, H. And Michel, P. : The second moment of the symmetric square L-functions. Ann. Acad. Sci. Fenn. Math. 26 (2001), 465-482.

[10] Iwaniec, H. : Topics in classical automorphic forms. Graduate Studies in Mathematics 17. American Mathematical Society, Providence, Rhode Island, 1997.

[11] Kowalski, E. And Michel, P. : The analytic rank of $J_{0}(q)$ and zeros of automorphic L-functions. Duke Math. J. 100 (1999), no. 3, 503-542.

[12] Kowalski, E. AND Michel, P. : Zeros of families of automorphic $L$-functions close to 1. Pacific J. Math. 207 (2002), no. 2, 411-431.

[13] LAU, Y.-K. : On the error term in an asymptotic formula for symmetric square L-function. Proc. Amer. Math. Soc. 132 (2004), no. 2, 317-323.

[14] Luo, W. : Values of symmetric square $L$-functions at 1. J. Reine Angew. Math. 506 (1999), 215-235.

[15] Luo, W. : Nonvanishing of $L$-values and the Weyl law. Ann. of Math. (2) 154 (2001), no. 2, 477-502.

[16] Michel, P. : Analytic number theory and families of automorphic $L$ functions. Lectures from the Graduate Summer School held in Park City, Summer 2002, IAS/Park City Mathematics Series, American Mathematical Society, Providence, RI, à paraître. 
[17] Ram Murty, M. : The analytic rank of $J_{0}(N)(\mathbf{Q})$. In Number theory (Halifax, NS, 1994), 263-277. CMS Conf. Proc. 15. Amer. Math. Soc., Providence, RI, 1995

[18] Royer, E. : Statistique de la variable aléatoire $L\left(\operatorname{sym}^{2} f, 1\right)$. Math. Ann. 321 (2001), no. 3, 667-687.

[19] Royer, E. : Interprétation combinatoire des moments négatifs des valeurs de fonctions $L$ au bord de la bande critique. Ann. Sci. École Norm. Sup. (4) 36 (2003), no. 4, 601-620.

[20] Serre, J.-P. : Cours d'arithmétique. Deuxième édition revue et corrigée. Le Mathématicien, No. 2. Presses Universitaires de France, Paris, 1977.

[21] Titchmarsh, E. C. : The theory of functions, seconde éd. Oxford University Press, Oxford, 1952.

[22] Titchmarsh, E. C. : The theory of the Riemann zeta-function, seconde éd. The Clarendon Press, Oxford University Press, New York, 1986.

[23] Wu, J. : Average values of symmetric square $L$-functions at the edge of the critical strip. Proc. Amer. Math. Soc. 131 (2003), no. 4, 1063-1070.

[24] Zagier, D. : Modular parametrizations of elliptic curves. Canad. Math. Bull. 28 (1985), no. 3, 372-384.

Recibido: 16 de diciembre de 2002

Revisado: 21 de enero de 2003

Emmanuel Royer

Université Montpellier II, I3M-UMR5030, CC051, F-34095 Montpellier cedex 5, France Université Paul Valéry, Montpellier III, MIAp,

F-34199 Montpellier cedex 5, France royer@darboux . math . univ-montp2 . fr emmanuel.royer@polytechnique.org

Jie $\mathrm{Wu}$

Institut Élie Cartan, UMR7502 UHP CNRS INRIA, Université Henri Poincaré, Nancy 1, F-54506 Vandouvre-lés-Nancy, France wujie@iecn.u-nancy.fr

Le premier auteur souhaite remercier les participants au groupe de travail d'Étienne Fouvry à l'Université Paris-Sud pour leurs riches commentaires qui ont abouti à ce travail. Le second auteur exprime sa gratitude à Étienne Fouvry pour son encouragement constant. 